Article

\title{
Effect of Alkyl Chain Length in POSS Nanocage on Non-Isothermal Crystallization Behavior of PCL/Amino-POSS Nanocomposites
}

\author{
M. Dolores Fernández ${ }^{(D)}$, Dailyn J. Guzmán, Johnny R. Ramos and M. Jesús Fernández *(D) \\ Department of Polymer Science and Technology, Faculty of Chemistry, University of the Basque \\ Country (UPV/EHU), P Manuel Lardizábal 3, 20018 San Sebastián, Spain; \\ mariadolores.fernandez@ehu.es (M.D.F.); daylyngm@yahoo.es (D.J.G.); johnram@hotmail.com (J.R.R.) \\ * Correspondence: mjesus.fernandez@ehu.es; Tel.: +34-943-01-5353
}

Received: 27 September 2019; Accepted: 17 October 2019; Published: 19 October 2019

check for updates

\begin{abstract}
The study of the non-isothermal crystallization behavior of polymers is of great importance due to the effect of degree of crystallinity and crystallization process on the polymer properties. The effect of aminopropylisobutyl polyhedral oligomeric silsesquioxane (APIBPOSS) and aminopropylisooctyl polyhedral oligomeric silsesquioxane (APIOPOSS) on poly( $\varepsilon$-caprolactone) (PCL) crystallization is studied by differential scanning calorimetry (DSC) under non-isothermal conditions and polarized optical microscopy (POM). The crystallization kinetics is analyzed using the Avrami and Mo models, and effective activation energies are evaluated by the Friedman isoconversional method. The results show that the compatibility between polyhedral oligomeric silsesquioxanes (POSS) and PCL and POSS loading affect the crystallization process. A higher crystallization temperature, a narrower size distribution of crystallite, and a faster crystallization rate are obtained in the presence of all the studied contents of APIBPOSS and at lower contents of APIOPOSS. At APIOPOSS contents higher than $2 \mathrm{wt} \%$, the crystallization temperature is lowered, the size distribution of crystallite is broadened, and the crystallization process is retarded. The presence of POSS leads to an increase in the number of nucleation sites, and a reduction in the size of the crystallite and the overall degree of crystallinity, as a result of the confinement of PCL chains caused by POSS nanoparticles.
\end{abstract}

Keywords: poly(e-caprolactone) nanocomposites; POSS nanoparticles; DSC; nonisothermal crystallization; kinetic

\section{Introduction}

Poly( $\varepsilon$-caprolactone) (PCL), a semi-crystalline aliphatic and biodegradable polyester, compatible with many types of polymers and having good biocompatibility, has been used in a wide range of applications including food packaging material, tissue engineering scaffolding, medical devices, and drug delivery systems [1,2]. However, some disadvantages such as slow crystallization rate, deficiencies in thermal stability, and poor mechanical properties have limited the applications of PCL. To overcome these problems, some nanofillers have been added to PCL, and significant improvement in the physical properties of PCL can be achieved with quite a small amount of nanofillers [3-7].

Polyhedral oligomeric silsesquioxanes (POSS) are one of the most extensively used nanoparticles in the preparation of hybrid polymer nanocomposites. The three-dimensional POSS molecules are characterized by a cubic core $\left(\mathrm{Si}_{8} \mathrm{O}_{12}\right)$, which is surrounded by organic groups linked to silicon atoms via covalent bonding. POSS molecules have been incorporated into organic polymers via copolymerization, grafting, or blending [8-10]. The incorporation of POSS molecules into some semi-crystalline polymers affects their crystallization behavior [11-13], and the physical and mechanical properties of a semi-crystalline polymer are strongly dependent on its crystallization and morphology. 
The crystallization process can proceed under either isothermal or non-isothermal conditions. The study of the non-isothermal crystallization kinetics of polymers is important since their processing often proceeds under non-isothermal conditions. Investigation of crystallization behavior can serve as a guide for process and application.

PCL is one of the polymers that have been blended with POSS. Various POSS derivatives have been used to prepare PCL-based nanocomposites [12-17]. Pan et al. [12] studied the morphology, isothermal, and non-isothermal melt crystallization of PCL/octaisobutyl-POSS nanocomposites. They found that the crystallization of PCL is enhanced by the presence of POSS and influenced by the POSS loading. They suggested that the presence of POSS might provide heterogeneous nucleation sites for the PCL crystallization while the aggregates of POSS might restrict large crystal growth of PCL. Miltner et al. [13] investigated the influence of the addition of aminopropylheptakis(isobutyl)-POSS into PCL on the thermal properties. The results showed that POSS nanoparticles barely nucleated the PCL crystallization, and therefore hardly affected the PCL semi-crystalline morphology and thermal behavior. Goffin et al. [14] reported the crystallization behavior and the thermomechanical properties of PCL/aminopropylheptakis(isobutyl)-POSS and PCL/aminopropylheptakis(isobutyl)-POSS-g-PCL. They saw that well-dispersed POSS nanoparticles acted as efficient nucleating sites, significantly increasing the crystallinity degree of PCL. Guan and Qiu [16] studied the isothermal crystallization, morphology, and dynamic mechanical properties of PCL in the PCL/octavinyl-POSS nanocomposites. The presence of ovi-POSS enhanced the overall isothermal melt crystallization rates of PCL in the nanocomposites, while the crystallization mechanism and crystal structure of PCL remained unchanged despite the POSS loading. Lee and Chang [17] reported the effect of trisilanolphenyl-POSS on the thermal and mechanical properties of PCL/POSS nanocomposites. They found that the degree of crystallinity of the PCL decreased with incorporation of POSS into PCL. To the best of our knowledge, there are no other reports indicating that the crystallization behavior of PCL blends with different POSS derivatives having different alkyl substituents. However, it has been reported that not only the POSS content but also the structure of the alkyl chains of POSS substituent groups have an effect on the crystallization kinetics of polymer/POSS nanocomposites [11,18-21].

The purpose of the present study is to ascertain the effect of the alkyl-chain length of the nonreactive organic substituents attached to the corner silicon atoms of two amine-functionalized POSS on crystallization behavior of PCL. Recently, we have reported the effect of the alkyl-chain length of these substituents attached to the corner silicon atoms of amino-derivative POSS on morphology, thermal, and mechanical properties of PCL/amino-POSS nanocomposites [22]. We found that the longer the alkyl chain, the better the extent of dispersion of POSS due to the greater compatibility with PCL chains. In the present study, aminopropylisobutyl polyhedral oligomeric silsesquioxane (APIBPOSS) and aminopropylisooctyl polyhedral oligomeric silsesquioxane (APIOPOSS) have been used to prepare PCL/APIBPOSS and PCL/APIOPOSS nanocomposites, respectively, with different POSS contents. The effects of the incorporation of amino-POSS derivatives on the non-isothermal melt crystallization kinetics and spherulitic morphology of PCL were investigated by differential scanning calorimetry (DSC) and polarized optical microscopy (POM). We have found that the POSS structure and content had an effect on the rate of crystallization and the overall amount of crystallinity of PCL. Lower degree of crystallinity and crystallization rate have been achieved using the POSS molecule, showing better compatibility with PCL compared to the less compatible POSS. The obtained material can be of semi-crystalline nature or almost amorphous depending on the POSS loading and the alkyl-chain length of the nonreactive organic substituents attached to the vertices of the POSS cage.

\section{Materials and Methods}

\subsection{Materials}

$\operatorname{PCL}\left(M_{n}=4.5 \times 10^{4} \mathrm{~g} \mathrm{~mol}^{-1}\right)$ was purchased from Aldrich (Munich, Germany). APIBPOSS and APIOPOSS were obtained from Hybrid Plastics (Hattiesburg, MS, USA), and used as received. 


\subsection{PCL/POSS Nanocomposite Preparation}

Nanocomposites with 2, 5, and $10 \mathrm{wt} \%$ POSS were obtained via solution and casting method as described elsewhere [22]. Both PCL and POSS were first dissolved in chloroform separately. Then they were mixed together and the mixture was sonicated for $3 \mathrm{~h}$. The mixture was poured into a dish to evaporate the solvent at room temperature for $24 \mathrm{~h}$. The obtained film was further kept in a vacuum at $50{ }^{\circ} \mathrm{C}$ for 2 days to remove the solvent completely. For comparison, the neat PCL film was prepared in the same way. The nanocomposites were named PCL/APIBPOSS-x and PCL/APIOPOSS-x, where $x$ denotes the weight percentage of POSS.

\subsection{Sample Characterization}

The non-isothermal crystallization characteristics of the pure PCL and PCL/POSS nanocomposites were measured with a differential scanning calorimeter (DSC-Q2000) from TA Instruments (New Castle, DE, USA). The temperature was calibrated with melting indium. All DSC measurements were performed under ultrapure nitrogen atmosphere on samples of about $5 \mathrm{mg}$, placed in aluminum pans. All specimens were first heated to $100{ }^{\circ} \mathrm{C}$ at $40{ }^{\circ} \mathrm{C} \mathrm{min}-1$, held for $5 \mathrm{~min}$ to erase previous thermal history and then were cooled to $-50^{\circ} \mathrm{C}$ at various cooling rates $(\phi)$ ranging from 5 to $25^{\circ} \mathrm{C} \mathrm{min}^{-1}$. After that, the samples were reheated from $-50{ }^{\circ} \mathrm{C}$ to $100{ }^{\circ} \mathrm{C}$ at $10^{\circ} \mathrm{C} \mathrm{min}^{-1}$ to evaluate the melting behavior. The peak crystallization temperature $\left(T_{\mathrm{cp}}\right)$ and heat of the crystallization $\left(\Delta H_{\mathrm{c}}\right)$ were obtained from the cooling scans after first heating, whereas the melting temperature $\left(T_{\mathrm{mp}}\right)$ and the heat of fusion $\left(\Delta H_{\mathrm{m}}\right)$ were obtained during second heating of the samples.

XRD patterns were recorded on a Bruker D8 Advance X-ray diffractometer (Karlsruhe, Germany) with a graphite monochromator, and the $\mathrm{Cu} \mathrm{K} \alpha$ radiation $(\lambda=0.154 \mathrm{~nm})$ operating at $40 \mathrm{kV} / 30 \mathrm{~mA}$.

The spherulitic morphology of neat PCL and the PCL/POSS nanocomposites was analyzed by polarized optical microscope (POM) on a Leitz Aristomet microscope (Wetzlar, Germany) equipped with a Mettler Toledo FP82HT hot stage controlled by a Mettler Toledo FP80 central processor (Mettler-Toledo SAE, Barcelona, Spain). The samples used for the POM analysis were placed between two glass slides and melted on the hot stage at $100{ }^{\circ} \mathrm{C}$ at $40{ }^{\circ} \mathrm{C} \mathrm{min}-1$, held there for 5 min to erase any thermal history, and then cooled to $25^{\circ} \mathrm{C}$ at $2{ }^{\circ} \mathrm{C} \mathrm{min}^{-1}$.

\section{Results}

\subsection{Non-Isothermal Crystallization and Melting Behavior}

The non-isothermal crystallization thermograms at various cooling rates, ranging from 5 to $25^{\circ} \mathrm{C}$ $\mathrm{min}^{-1}$, for neat PCL, PCL/APIBPOSS, and PCL/APIOPOSS nanocomposites are shown in Figure 1. From these crystallization exotherms, the temperature at $1 \%$ relative crystallinity $\left(T_{\mathrm{c} 0.01}\right)$, the temperature at $99 \%$ relative crystallinity $\left(T_{\mathrm{c} 0.99}\right)$, and the temperature at the maximum crystallization rate $\left(T_{\mathrm{cp}}\right)$ were obtained. $T_{c 0.01}$, used to represent the beginning of the crystallization process, $T_{\mathrm{c} 0.99}$, used to represent the ending of the crystallization process for PCL and nanocomposites, are reported in Table 1, while Figure 2 shows $T_{\mathrm{cp}}$ as a function of $\phi$.

As the cooling rate increases, the crystallization exotherm broadens and shifts to lower temperatures for all samples, including neat PCL and POSS-based nanocomposites. With increasing of cooling rate, $T_{\mathrm{c} 0.01}, T_{\mathrm{cp}}$, and $T_{\mathrm{c} 0.99}$ shift gradually to the lower temperatures, indicating that the lower the cooling rate is, the earlier the crystallization starts. At a higher cooling rate, the activation of nuclei occurs at lower temperatures, whereas when the samples are cooled at lower scanning rates, there is enough time to activate nuclei and crystallization occurs at higher temperatures. When cooled at a low rate, the melt polymer chains have enough response time to crystallize, whereas when the samples are cooled fast, the motion of polymer molecules are not able to follow crystallization temperature. 

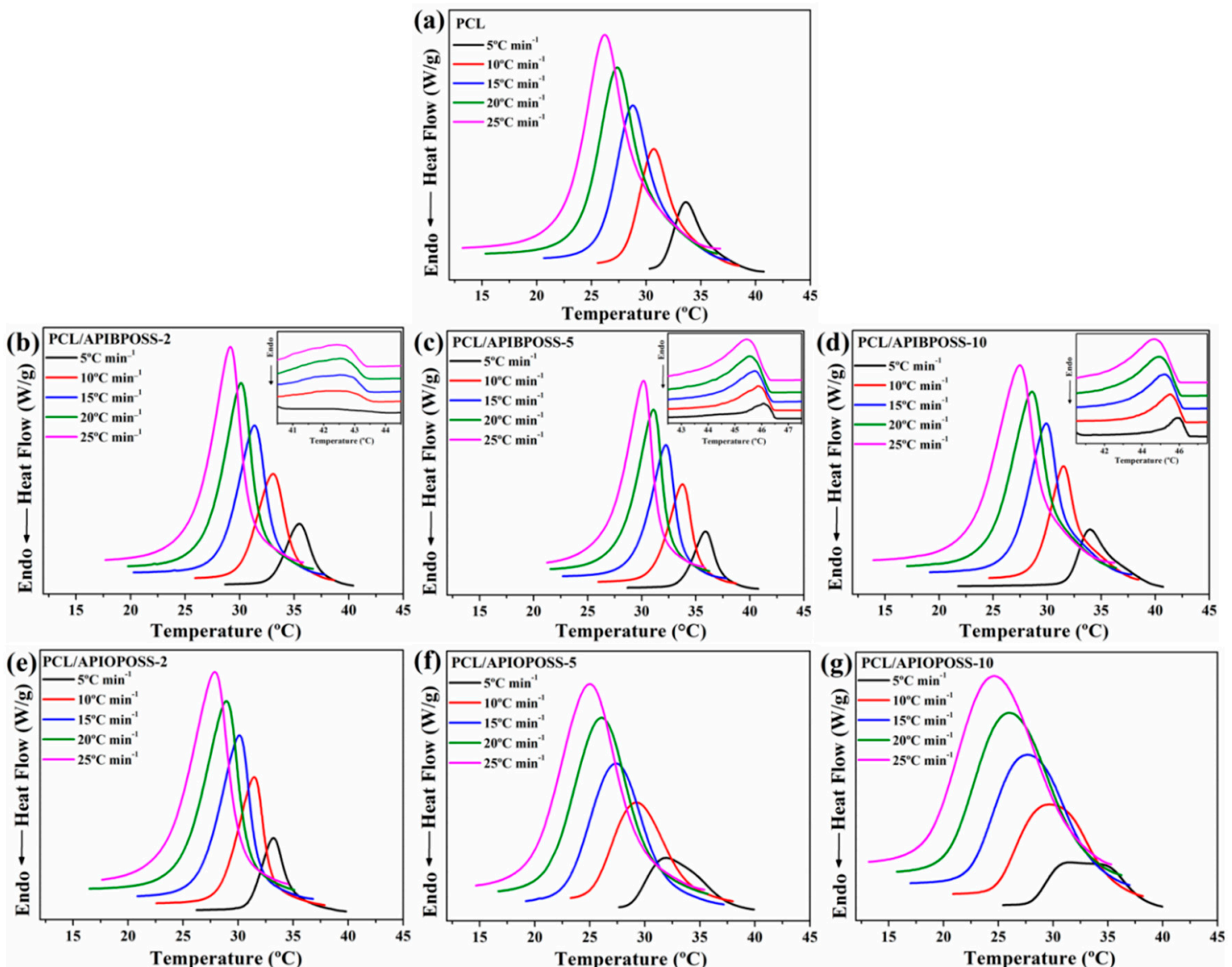

Figure 1. DSC traces of (a) PCL, (b-d) PCL/APIBPOSS nanocomposites, and (e-g) PCL/APIOPOSS nanocomposites during cooling at various cooling rates.

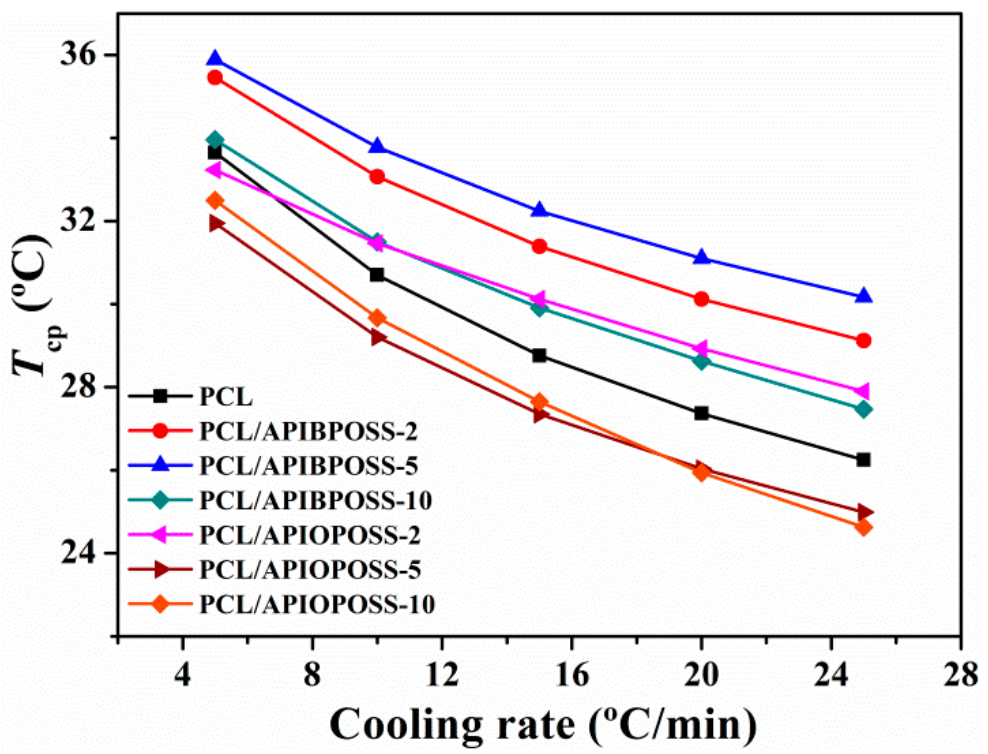

Figure 2. Crystallization peak temperatures as a function of cooling rate for PCL and PCL/polyhedral oligomeric silsesquioxanes (POSS) nanocomposites. 
Table 1. Differential scanning calorimetry (DSC) results of pure poly( $\varepsilon$-caprolactone) (PCL), PCL/aminopropylisooctyl polyhedral oligomeric silsesquioxanes (APIBPOSS), and PCL/aminopropylisobutyl polyhedral oligomeric silsesquioxanes (APIOPOSS).

\begin{tabular}{|c|c|c|c|c|c|c|c|c|c|c|}
\hline Sample & $\begin{array}{c}\phi \\
\left({ }^{\circ} \mathrm{C} \mathrm{min}^{-1}\right)\end{array}$ & $\begin{array}{c}T_{\mathrm{c} 0.01} \\
\left({ }^{\circ} \mathrm{C}\right)\end{array}$ & $\begin{array}{c}T_{\mathrm{c} 0.99} \\
\left({ }^{\circ} \mathrm{C}\right)\end{array}$ & $\begin{array}{c}\Delta t_{\mathrm{c}} \\
(\mathrm{min})\end{array}$ & $\begin{array}{c}\Delta H_{\mathrm{c}} \\
\left(\mathrm{J} \mathrm{g}^{-1}\right)\end{array}$ & $\begin{array}{l}T_{\mathrm{mp}} \\
\left({ }^{\circ} \mathrm{C}\right)\end{array}$ & $\begin{array}{c}T_{\mathrm{mp}}-T_{\mathrm{cp}} \\
\quad\left({ }^{\circ} \mathrm{C}\right)\end{array}$ & $\begin{array}{c}\Delta H_{\mathrm{m}} \\
\left(\mathrm{J} \mathrm{g} \mathrm{g}^{-1}\right)\end{array}$ & $\begin{array}{c}\text { CRC } \\
\left(\min ^{-1}\right)\end{array}$ & $\begin{array}{c}\text { CRP } \\
\left({ }^{\circ} \mathrm{C}^{-1}\right)\end{array}$ \\
\hline \multirow{5}{*}{ PCL } & 5 & 38.92 & 31.38 & 1.508 & 37.34 & 55.96 & 22.31 & 40.94 & \multirow{5}{*}{3.270} & \multirow{5}{*}{0.0854} \\
\hline & 10 & 36.70 & 27.38 & 0.932 & 37.20 & 55.65 & 24.95 & 40.22 & & \\
\hline & 15 & 35.41 & 23.86 & 0.770 & 37.45 & 55.45 & 26.69 & 40.82 & & \\
\hline & 20 & 34.33 & 20.43 & 0.695 & 37.43 & 55.34 & 27.97 & 40.09 & & \\
\hline & 25 & 33.73 & 18.35 & 0.615 & 37.45 & 55.20 & 28.55 & 40.20 & & \\
\hline \multirow{5}{*}{ PCL/APIBPOSS-2 } & 5 & 39.03 & 31.48 & 1.510 & 36.85 & 56.05 & 20.59 & 41.08 & \multirow{5}{*}{3.806} & \multirow{5}{*}{0.1260} \\
\hline & 10 & 36.97 & 28.66 & 0.831 & 35.72 & 55.75 & 22.68 & 41.50 & & \\
\hline & 15 & 35.68 & 24.89 & 0.719 & 36.25 & 55.57 & 24.18 & 40.80 & & \\
\hline & 20 & 34.55 & 23.39 & 0.558 & 36.40 & 55.47 & 25.35 & 41.02 & & \\
\hline & 25 & 33.61 & 21.59 & 0.481 & 36.31 & 55.33 & 26.21 & 40.28 & & \\
\hline \multirow{5}{*}{ PCL/APIBPOSS-5 } & 5 & 39.21 & 31.83 & 1.476 & 35.04 & 56.10 & 20.21 & 39.29 & \multirow{5}{*}{4.171} & \multirow{5}{*}{0.1684} \\
\hline & 10 & 37.06 & 29.04 & 0.802 & 35.54 & 55.82 & 22.04 & 37.24 & & \\
\hline & 15 & 35.74 & 26.28 & 0.631 & 35.95 & 55.68 & 23.44 & 37.09 & & \\
\hline & 20 & 34.43 & 24.67 & 0.488 & 35.74 & 55.56 & 24.46 & 37.93 & & \\
\hline & 25 & 33.63 & 23.58 & 0.402 & 35.77 & 55.51 & 25.34 & 36.98 & & \\
\hline \multirow{5}{*}{ PCL/APIBPOSS-10 } & 5 & 39.37 & 27.23 & 2.428 & 33.29 & 56.39 & 22.43 & 38.64 & \multirow{5}{*}{3.334} & \multirow{5}{*}{0.1029} \\
\hline & 10 & 36.93 & 27.44 & 0.949 & 32.76 & 56.18 & 24.68 & 37.87 & & \\
\hline & 15 & 35.59 & 23.51 & 0.805 & 32.86 & 56.13 & 26.22 & 37.52 & & \\
\hline & 20 & 34.30 & 21.19 & 0.656 & 32.94 & 55.95 & 27.32 & 37.54 & & \\
\hline & 25 & 33.43 & 18.54 & 0.596 & 32.67 & 55.75 & 28.28 & 37.25 & & \\
\hline \multirow{5}{*}{ PCL/APIOPOSS-2 } & 5 & 38.13 & 29.52 & 1.722 & 37.60 & 56.13 & 22.90 & 38.46 & \multirow{5}{*}{4.655} & \multirow{5}{*}{0.1463} \\
\hline & 10 & 35.84 & 26.29 & 0.955 & 36.91 & 55.74 & 24.28 & 38.62 & & \\
\hline & 15 & 34.44 & 24.14 & 0.687 & 36.81 & 55.6 & 25.48 & 37.95 & & \\
\hline & 20 & 33.03 & 21.26 & 0.588 & 36.63 & 55.43 & 26.50 & 37.96 & & \\
\hline & 25 & 32.21 & 20.56 & 0.466 & 36.41 & 55.34 & 27.44 & 38.36 & & \\
\hline \multirow{5}{*}{ PCL/APIOPOSS-5 } & 5 & 38.25 & 27.41 & 2.168 & 36.74 & 56.02 & 24.07 & 37.03 & \multirow{5}{*}{3.512} & \multirow{5}{*}{0.0897} \\
\hline & 10 & 35.83 & 24.56 & 1.127 & 36.59 & 55.72 & 26.51 & 37.10 & & \\
\hline & 15 & 34.25 & 21.54 & 0.847 & 36.32 & 55.50 & 28.15 & 37.47 & & \\
\hline & 20 & 33.16 & 19.28 & 0.694 & 36.57 & 55.28 & 29.24 & 37.34 & & \\
\hline & 25 & 32.39 & 17.82 & 0.583 & 36.53 & 55.12 & 30.13 & 37.15 & & \\
\hline \multirow{5}{*}{ PCL/APIOPOSS-10 } & 5 & 38.52 & 28.09 & 2.086 & 36.87 & 55.78 & 23.28 & 37.66 & \multirow{5}{*}{3.236} & \multirow{5}{*}{0.0814} \\
\hline & 10 & 36.36 & 24.44 & 1.192 & 36.52 & 55.4 & 25.73 & 37.78 & & \\
\hline & 15 & 34.92 & 21.41 & 0.901 & 36.22 & 55.2 & 27.55 & 38.36 & & \\
\hline & 20 & 33.93 & 19.33 & 0.730 & 36.24 & 55.05 & 29.11 & 37.54 & & \\
\hline & 25 & 32.94 & 17.08 & 0.634 & 35.75 & 54.98 & 30.35 & 37.19 & & \\
\hline
\end{tabular}

The DSC curves of the PCL/APIBPOSS nanocomposites, in addition to the exothermic peak corresponding to the PCL crystallization, exhibit another small exothermic transition in the temperature range $41-47^{\circ} \mathrm{C}$ (inset of Figure $1 \mathrm{~b}-\mathrm{d}$ ) that corresponds to the APIBPOSS crystal formation. As we previously reported [22], APIBPOSS crystallizes and agglomerates in PCL matrix due to the POSS-POSS interactions. Due to the amorphous nature of APIOPOSS, the DSC curves of PCL/APIOPOSS composites exhibit only the exothermic crystallization peak of PCL.

The incorporation of APIBPOSS into the PCL matrix leads to an increase in $T_{\mathrm{cp}}$ of the PCL/APIBPOSS nanocomposites (Figure 2). The difference between $T_{\mathrm{cp}}$ values of neat PCL and those of APIBPOSS-based nanocomposites increases as cooling rate increases. However, the addition of APIOPOSS to the PCL matrix leads to an increase in $T_{\mathrm{cp}}$ of the nanocomposite with $2 \mathrm{wt} \%$ POSS loading and to a decrease in $T_{\mathrm{cp}}$ with POSS loadings greater than $2 \mathrm{wt} \%$. These results suggest that APIBPOSS increases the crystallization rate of PCL, while the crystallization rate of PCL upon the addition of APIOPOSS depends on POSS loading. The nucleation of PCL crystals is retarded by the addition of APIOPOSS nanoparticles at a concentration equal or higher than $5 \mathrm{wt} \%$. The addition of POSS nanoparticles as fillers into PCL shifts the crystallization peak temperature to higher values because spherulites are created around the added particles and they act as nucleation centers, making the molecular chains of PCL easier to crystallize and increasing the crystallization rate. 
The total crystallization time $\left(\Delta t_{\mathrm{c}}\right)$ for neat PCL and the nanocomposite is calculated as

$$
\Delta t_{c}=\frac{T_{0.01}-T_{0.99}}{\phi}
$$

where $\phi$ is the non-isothermal constant cooling rate, which decreases as cooling rate increases (Table 1) for both pristine PCL and PCL/POSS nanocomposites. This result implies that as the cooling rate increases, the crystallization process becomes faster and the process takes less time to complete. For example, the crystallization period of PCL sample at a cooling rate of $5^{\circ} \mathrm{C} \mathrm{min}^{-1}$ is about $1.51 \mathrm{~min}$, whereas it shortens to $0.69 \mathrm{~min}$ when the cooling rate is increased to $20^{\circ} \mathrm{C} \mathrm{min}^{-1}$. Crystallization of PCL and its nanocomposites occurs at a higher temperature and over a larger time with decreasing cooling rate, suggesting that the crystallization is controlled by nucleation process [23]. In addition, the values of $\Delta t_{\mathrm{c}}$ are reduced for the PCL/APIBPOSS nanocomposites with 2 and $5 \mathrm{wt} \%$ POSS as compared to the pure PCL for all cooling rates, whereas the PCL/APIOPOSS nanocomposite with $2 \mathrm{wt}$ $\%$ POSS exhibits shorter crystallization time than PCL at cooling rates higher than $5{ }^{\circ} \mathrm{C} \mathrm{min}^{-1}$. The lowered value for the $\Delta t_{\mathrm{c}}$ implies that the time required for the crystallization process to be completed is somewhat lower for the nanocomposites, and suggests a decreasing time for the growth of the crystals in the presence of POSS and possibly indicates the effect of significantly increased nucleation density in the POSS nanocomposites. POSS nanoparticles can act as nucleation centers, favoring the process and increasing the speed of crystal formation.

The width at half height of the exothermic peak of crystallization, $\Delta W$, is another useful parameter to describe the non-isothermal crystallization behavior of PCL and its nanocomposites. The $\Delta W$ denotes the distribution of the forming crystal dimensions, that is, the smaller the $\Delta W$, the narrower the distribution. It can be seen (Figure 3) that the distribution of the crystal dimensions is in the order of $\Delta W_{\text {PCL/APIBPOSS }}<\Delta W_{\mathrm{PCL}}<\Delta W_{\mathrm{PCL} / \mathrm{APIOPOSS}}$ at a given cooling rate. The $\Delta W$ increases with the increasing cooling rate for PCL and PCL/POSS nanocomposite, which suggests that increasing supercooling results in a broader distribution of the crystal dimensions. Corresponding to the same cooling rate, the crystallite size distribution of the PCL/APIBPOSS-2 nanocomposite is the most uniform.

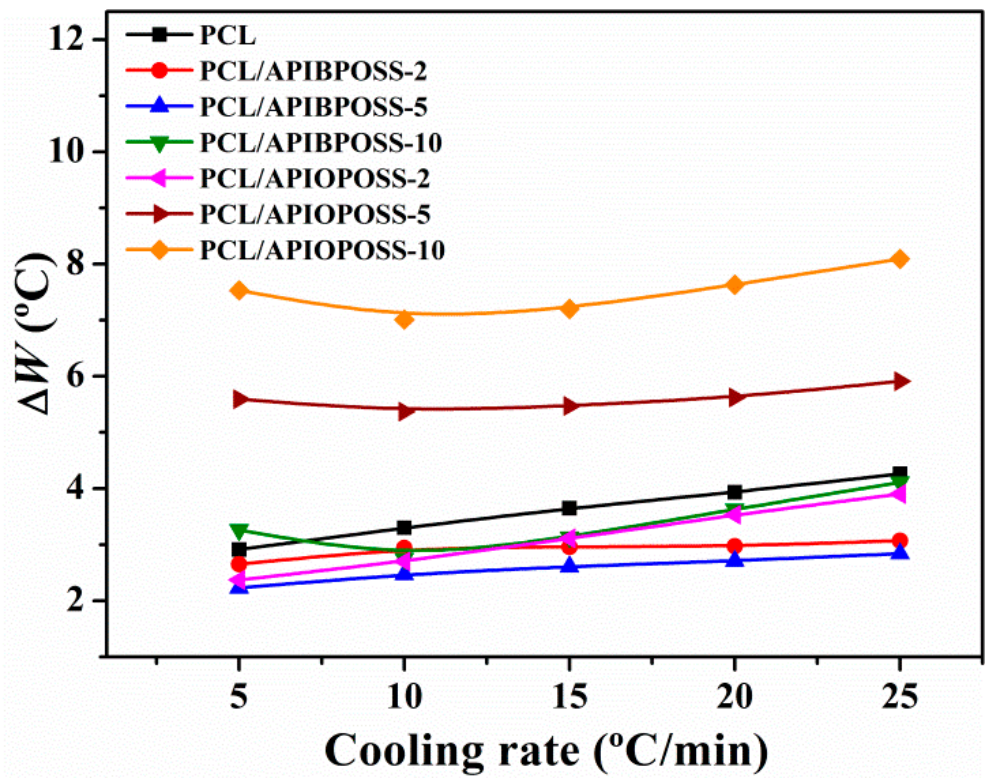

Figure 3. Crystallization peak width at half maximum as a function of cooling rate for PCL and PCL/POSS nanocomposites.

From the DSC thermograms of the cooling process, the temperature at the intercept of the tangent at the base line and the high temperature side of the exotherm peak was obtained $\left(T_{k}\right)[24,25]$. The parameter $\left(T_{\mathrm{k}}-T_{\mathrm{cp}}\right)$ is a measure of the overall rate of crystallization; lower values of $\left(T_{\mathrm{k}}-T_{\mathrm{cp}}\right)$ suggest 
faster crystallization. From Figure 4, it can be seen that the nanocomposites containing 5 and $10 \mathrm{wt} \%$ APIOPOSS exhibit the slower crystallization rate, while the nanocomposites containing APIBPOSS and $2 \mathrm{wt} \%$ APIOPOSS exhibit faster crystallization as compared with neat PCL.

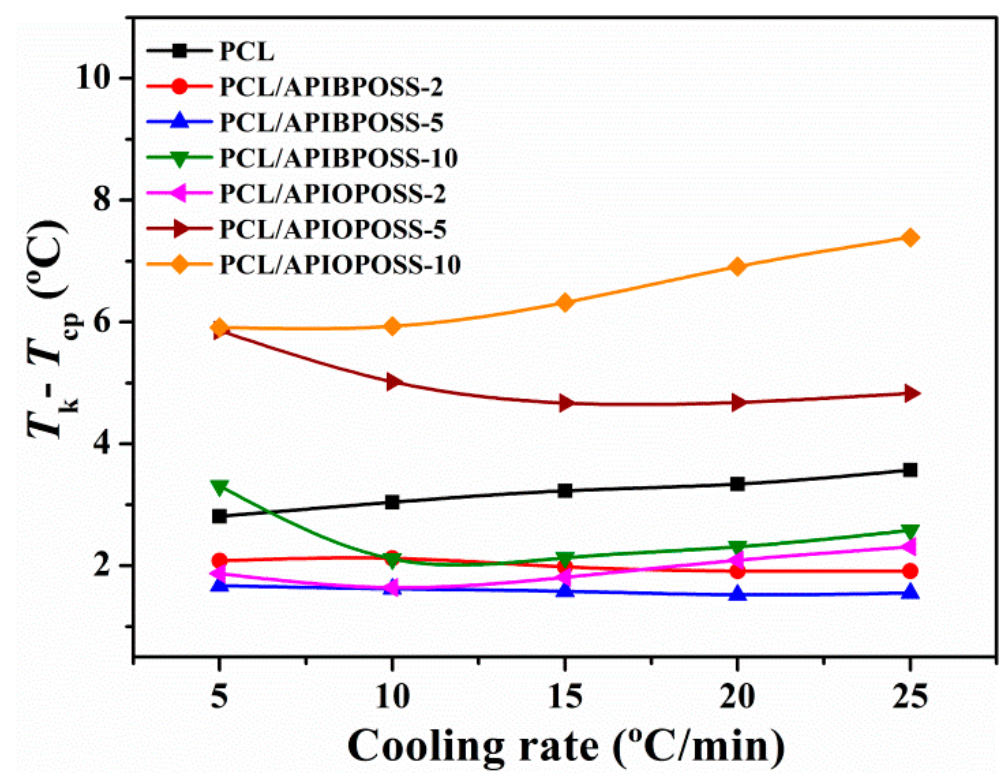

Figure 4. $\left(T_{\mathrm{k}}-T_{\mathrm{cp}}\right)$ parameter as a function of cooling rate for PCL and PCL/POSS nanocomposites.

Table 1 also shows the heat of crystallization $\left(\Delta H_{\mathrm{c}}\right)$. It is observed that the crystallization enthalpy is affected by both the addition of POSS and cooling rate. The values of $\Delta H_{\mathrm{c}}$ obtained for both neat PCL and PCL/POSS nanocomposite decrease with increasing the cooling rate, and the addition of POSS leads to a decrease in $\Delta H_{\mathrm{C}}$, indicating that the crystallinity of PCL is affected by the incorporation of POSS.

The thermograms for the second heating process are shown in Figure 5. The melting temperature $\left(T_{\mathrm{mp}}\right)$ and the melting enthalpy $\left(\Delta H_{\mathrm{m}}\right)$ were evaluated from these curves. The results of the melting behavior following each of the non-isothermal crystallization experiments are reported in Table 1.

The melting endotherm of neat PCL exhibits a shoulder on the higher temperature side, and it becomes more obvious as the cooling rate increases. Similar behavior is observed for the PCL/POSS nanocomposites. The melting peak and the shoulder at the higher temperature shift gradually toward lower temperature with increasing cooling rate, and the endothermic curves broaden. More perfect crystals are formed when the melt is cooled at low cooling rate, resulting in a higher melting peak. When the cooling rate is increased, the polymer chains could not be correspondingly rearranged into the lattice in such a short time, resulting in less perfect crystals that melt at a lower temperature. These crystals, then during heating recrystallize and reorganize into more perfect and stable crystals that melt at higher temperatures. The double endothermic peak can be attributed to the melting of the crystals that recrystallize during the heating process.

The $T_{\mathrm{mp}}$ of the peaks at the lower and higher temperatures (Table 1 and Figure 6) for the PCL/APIBPOSS and PCL/APIOPOSS nanocomposites are almost unchanged in comparison with the neat PCL at all cooling rates. The $\Delta H_{\mathrm{m}}$ value for PCL is insensitive to the cooling rate at the studied range, whereas for PCL/APIBPOSS and PCL/APIOPOSS nanocomposites this value decreases with increasing the cooling rate, and the addition of POSS leads to a decrease in $\Delta H_{\mathrm{m}}$ as compared with neat PCL. 

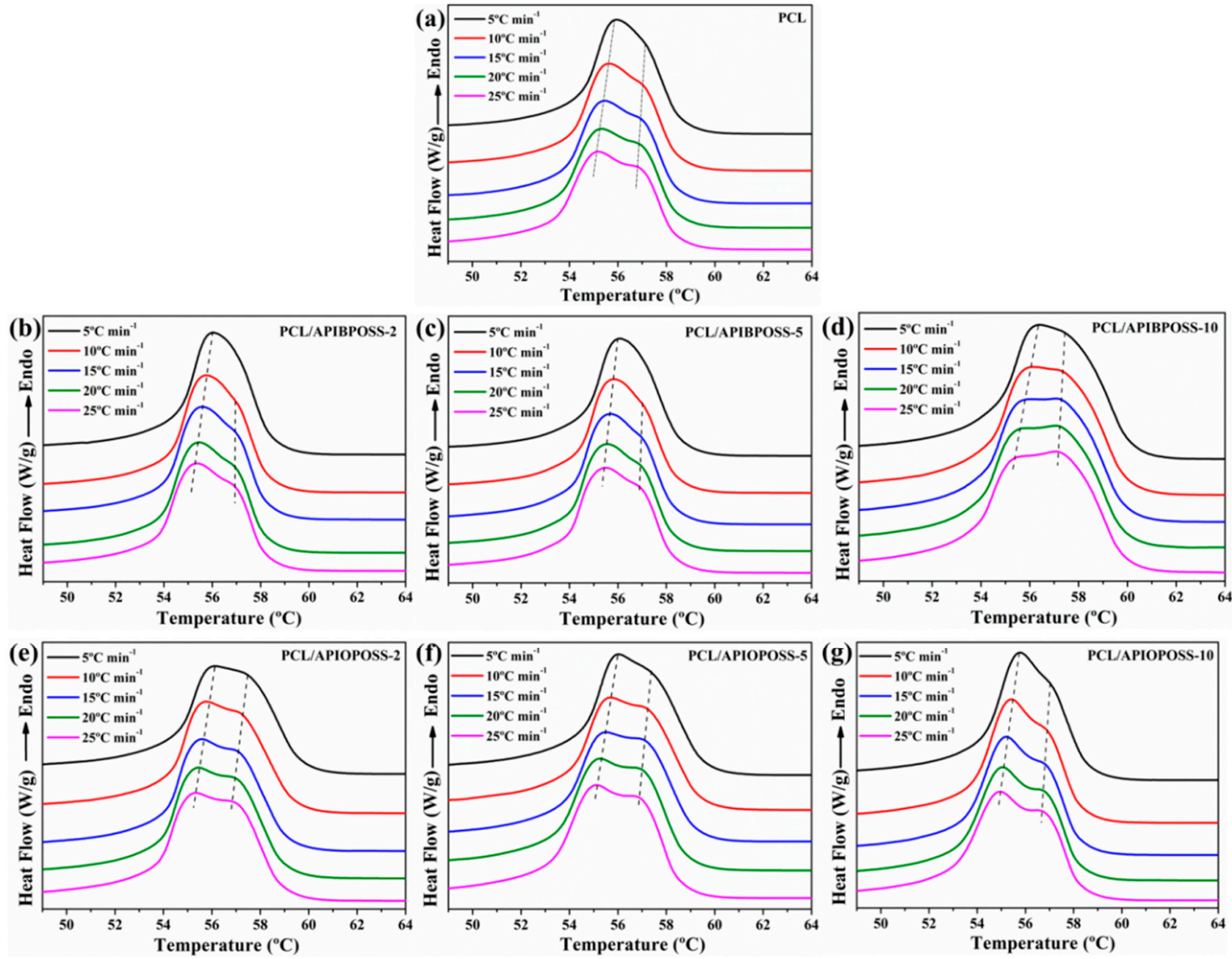

Figure 5. DSC thermograms of melting of (a) PCL, (b-d) PCL/APIBPOSS nanocomposites, and (e-g) PCL/APIOPOSS nanocomposites obtained during heating at $10{ }^{\circ} \mathrm{C} \mathrm{min}-1$ after non-isothermal crystallization at the indicated cooling rates.
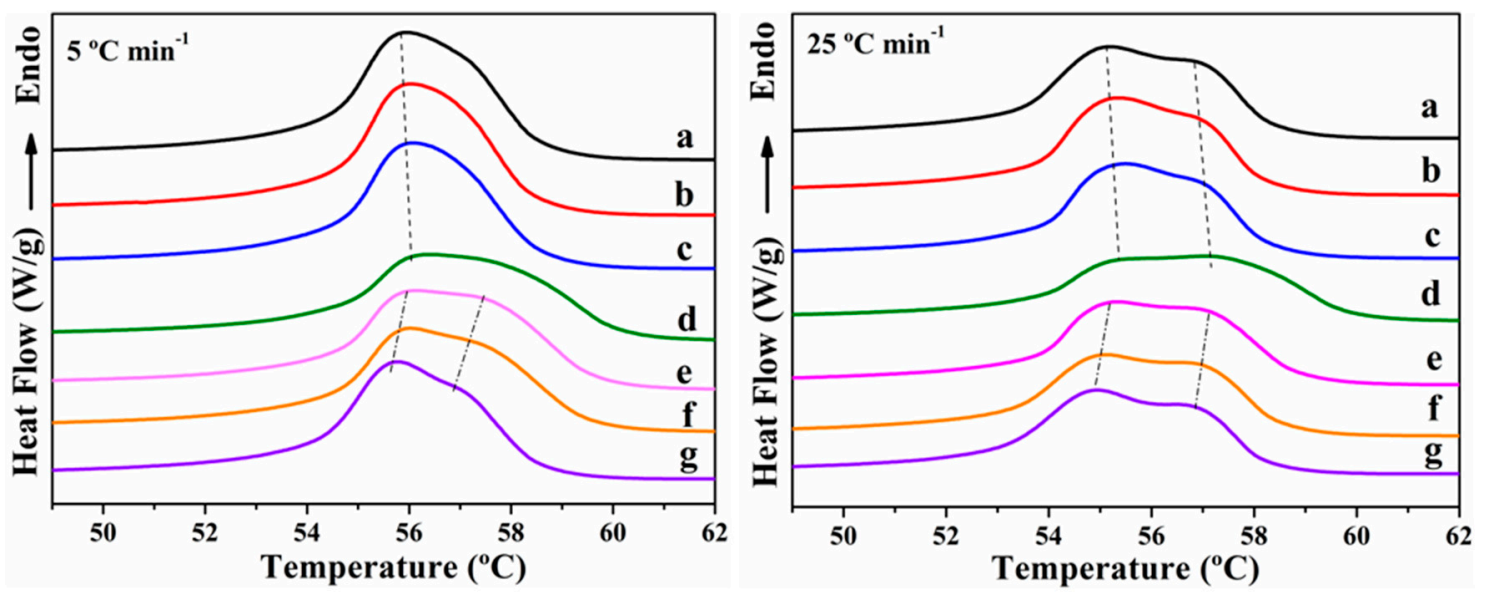

Figure 6. DSC thermograms of melting of (a) PCL, (b) PCL/APIBPOSS-2, (c) PCL/APIBPOSS-5, (d) PCL/APIBPOSS-10, (e), PCL/APIOPOSS-2 (f) PCL/APIOPOSS-5, and (g) PCL/APIOPOSS-10 nanocomposites obtained during heating at $10{ }^{\circ} \mathrm{C} \mathrm{min}{ }^{-1}$ after non-isothermal crystallization at the indicated cooling rates. 
Figure 7 shows the extent of crystallinity $\left(X_{c}\right)$ calculated by the following equation:

$$
X_{c}=\left[\frac{\Delta H_{m}}{\Delta H_{m}^{0} \times\left(1-\frac{\% w t_{\text {filler }}}{100}\right)}\right] \times 100
$$

where $\Delta H_{\mathrm{m}}$ is the specific heat of fusion of the sample, $\Delta H_{m}^{0}$ is the enthalpy of fusion of a perfect PCL crystal (142 J/g [26]), and \% $w t_{\text {filler }}$ is the total weight percentage of nanofiller.

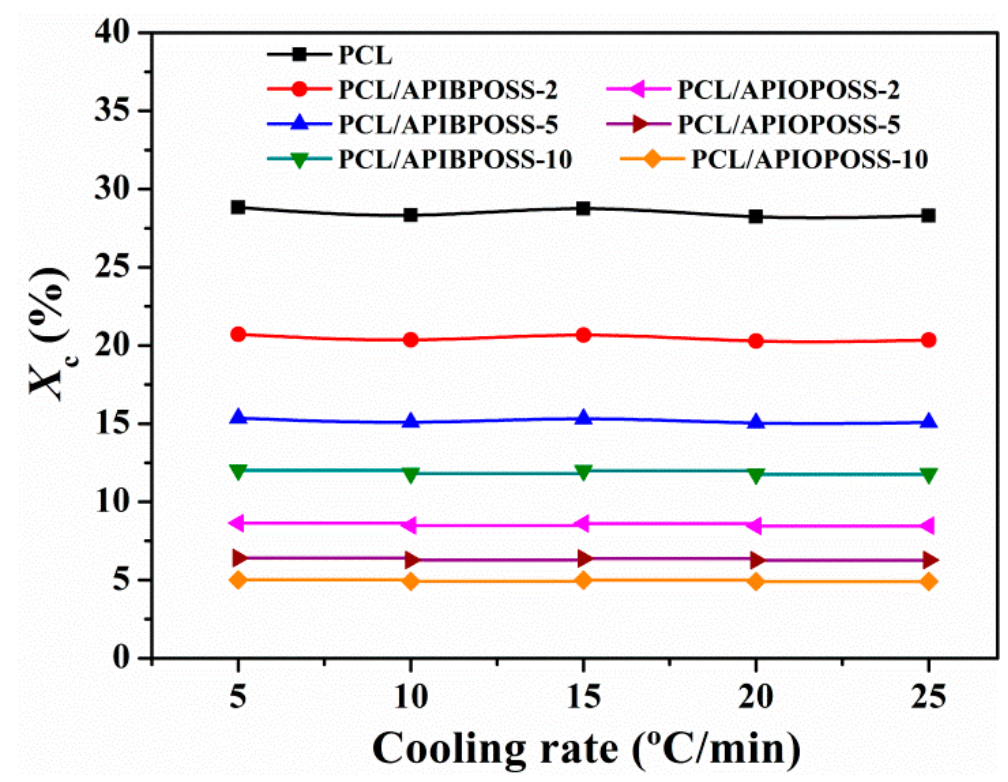

Figure 7. Degree of crystallinity as a function of cooling rate for PCL and PCL/POSS nanocomposites.

The crystallinity of PCL and its POSS-based nanocomposites are independent of the cooling rate. The addition of APIBPOSS and APIOPOSS in the PCL results in a significant reduction in the crystallinity, and the higher the POSS loading the lower the degree of crystallinity. In the case of APIBPOSS nanocomposites, at $10 \mathrm{wt} \%$ APIBPOSS content, the crystallinity drops from 28 to $12 \%$ as compared to neat PCL. A more pronounced reduction is observed for APIOPOSS-based nanocomposites: in the presence of $10 \mathrm{wt} \%$ APIOPOSS, the crystallinity falls to $5 \%$.

The crystallinity of the polymer matrix in the presence of nanofillers can increase, decrease, or remain similar depending on the effect of the filler. If the nanofiller acts as a nucleating agent, either an enhancement or maintenance of crystallinity is expected, whereas a decrement is observed if the nanofiller hinders the mobility of the polymer chains. The decrease in crystallinity is not the expected behavior for APIBPOSS-based nanocomposites since the above results suggest that it acts as nucleating agent. The liquid APIOPOSS exhibits higher compatibility with PCL than the crystalline solid APIBPOSS, which was attributed to the more thermodynamically favorable interactions between PCL and APIOPOSS [22]. The better compatibility leads to better dispersion; hence, smaller size aggregates were found in the case of PCL/APIOPOSS blends. Crystalline aggregates of APIBPOSS molecules are present in PCL/APIBPOSS nanocomposites. The incorporation of APIOPOSS results in a retarded nucleation of PCL crystals at POSS contents higher than $2 \mathrm{wt} \%$, and a remarkable reduction in the degree of crystallinity. Pracella et al. [18] reported similar results in their study of the crystallization behavior of nanocomposites of isotactic polypropylene (PP) with POSS having different alkyl substituents. They found that the liquid isooctyl-POSS displayed a very fine filler dispersion compared with the crystalline solids octamethyl-POSS and octaisobutyl-POSS, lower crystallization rate than PP and other PP/POSS nanocomposites, and no nucleation activity. They ascribed their results to the high dispersion of isooctyl-POSS as liquid phase component. Heeley et al. [20] prepared 
polyethylene/POSS nanocomposites bearing different long linear alkyl groups, all POSS molecules being crystalline solids. They observed that there was some aggregation of POSS crystals in the polymer matrix but, as the alkyl-chain length increased, the POSS dispersed into the amorphous domains of the polyethylene. From the crystallization behavior of their blends, they conclude that regardless of the dispersion degree in the polymer matrix, all POSS molecules acted as nucleating agents, and increased the crystallinity and crystallization kinetics when compared with pure polyethylene. Taking into account the results of our study and the studies above mentioned, it could be concluded that the liquid nature of APIOPOSS and better compatibility with PCL are responsible for the different crystallization behavior of the PCL/APIOPOSS nanocomposites compared to those containing APIBPOSS. The reduction in the overall crystallinity with increasing POSS content can be explained by the formation of larger aggregates, which hinder the transport of the polymer chains from the melt toward the crystallization growth front.

Nucleation and retarded polymer chain mobility are the two factors that influence and compete in the crystallization. The nucleation effect prevails at low amino-POSS loading, increasing the crystallization rate, whereas as the POSS content increases, the retarded mobility of the polymer chains becomes more important and dominates over the nucleation, and the rate of crystallization decreases.

Under non-isothermal conditions, the crystallization process of PCL is hindered by the incorporation of both amino-POSS derivatives, with the hindrance more pronounced in the presence of APIOPOSS.

For non-isothermal crystallization, the relative degree of crystallinity, $X_{T}$, which is a function of temperature, is defined as

$$
X_{T}=\frac{\int_{T_{0}}^{T}\left(d H_{\mathcal{c}} / d T\right) d T}{\int_{T_{0}}^{T_{\infty}}\left(d H_{\mathcal{c}} / d T\right) d T}
$$

where $T_{0}$ and $T_{\infty}$ represent the initial and the end of crystallization temperature, respectively; $T$ is any temperature in the crystallization process and $d H_{\mathrm{c}}$ represents the differential crystallization enthalpy change in temperature range of $d T$.

Figure 8 shows the relative crystallinity $\left(X_{T}\right)$ as a function of temperature for PCL, PCL/APIBPOSS, and PCL/APIOPOSS nanocomposites. It can be seen that all curves in Figure 8 have approximately the same reversed sigmoidal shapes. The higher the cooling rate, the lower the temperature to initiate the crystallization. There is no enough time to activate nuclei at higher temperatures when crystallized at higher cooling rates, and nucleation occurs at lower temperatures.

The data of $X_{T}$ can be transformed into $X_{t}$ using the following equation:

$$
t=\frac{T_{0}-T}{\phi}
$$

where $T$ is the temperature at the crystallization time $t$, and $\phi$ is the cooling rate. The plots of $X_{t}$ as a function of $t$ for neat PCL, PCL/APIBPOSS, and PCL/APIOPOSS nanocomposites are presented in Figure 9. All the curves have similar sigmoidal shapes, and the lower the cooling rate is, the larger the time range over which the crystallization occurs, implying that the crystallization is controlled by the nucleation process. Crystallization of PCL and PCL/POSS nanocomposites occurs at a higher temperature and is completed in a longer time under a lower cooling rate. Three distinct periods for these S-shaped crystallization curves can be observed: an induction period corresponding to the primary nucleation process that takes place in the homogeneous melt, followed by a rapid increase of the crystallization where the crystal growth occurs at the crystal-melt interface, and ultimately a constant value of crystallinity is reached because of the spherulite impingement in the later stage of crystallization. During the early stage, fast primary crystallization happened, and in the later stage slow secondary crystallization occurred. At higher cooling rates, the transition between the primary and secondary crystallization regions was less pronounced. 


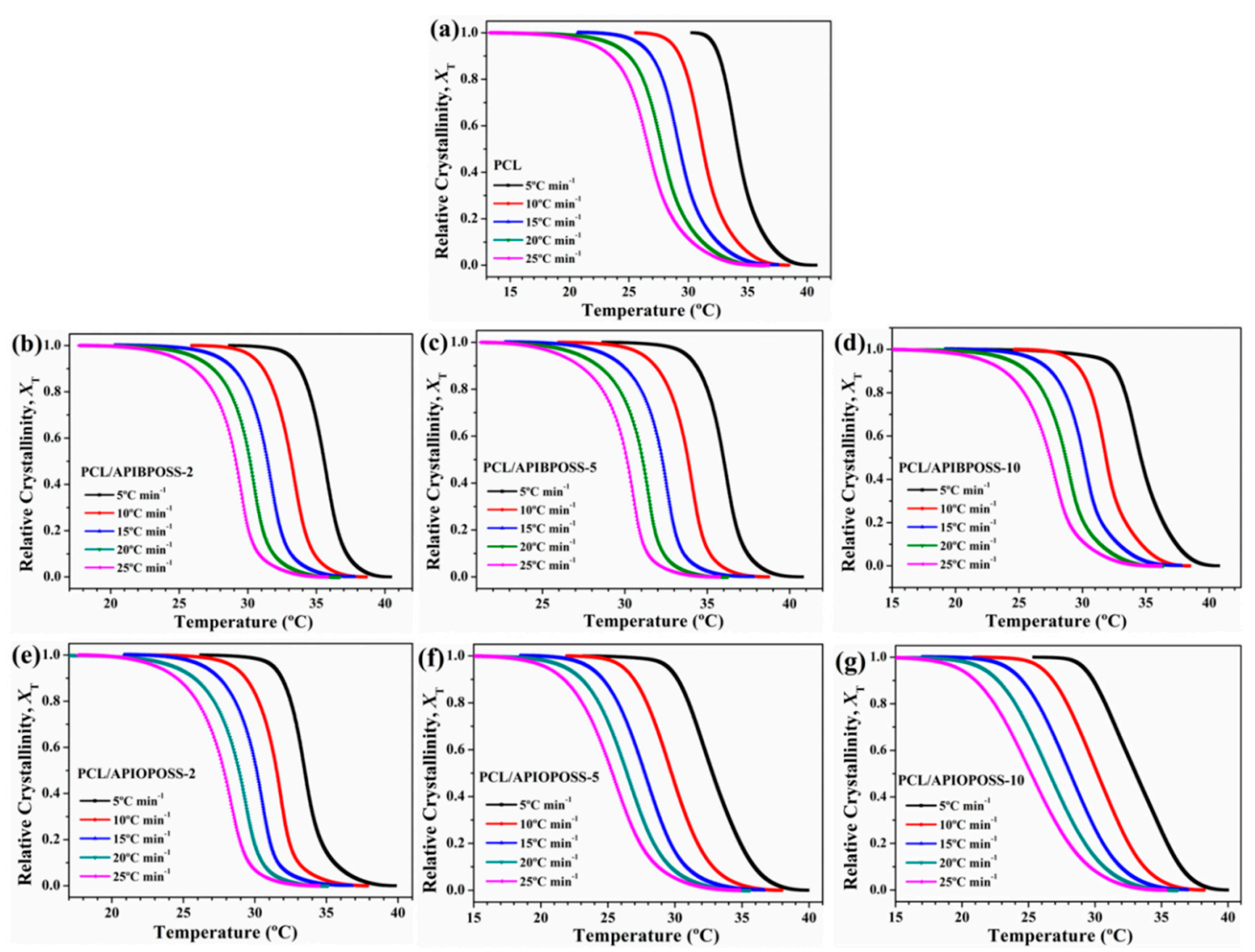

Figure 8. Development of relative crystallinity with temperature for non-isothermal crystallization of: (a) PCL, (b-d) PCL/APIBPOSS nanocomposites, (e-g) PCL/APIOPOSS nanocomposites at various cooling rates.

From Figure 9, the half time of crystallization $\left(t_{1 / 2}\right)$ can be taken directly, which is the change in time from the beginning of crystallization to the time at which $X_{t}$ is $50 \%$. The inverse value of $t_{1 / 2}$ denotes the bulk crystallization rate and the lower the $1 / t_{1 / 2}$ value, the slower the crystallization. Figure 10 presents the values of reciprocal of crystallization halftime as a function of cooling rate.

The $1 / t_{1 / 2}$ values depend on the cooling rate, increasing with increasing cooling rate, indicating that non-isothermal crystallization rate of PCL and nanocomposite becomes faster with increasing cooling rate. When cooling occurs at a higher rate, quick freezing of chain mobility takes place. The crystallization rate is higher for all PCL/APIBPOSS samples and PCL/APIOPOSS at 2 wt \% loading than neat PCL, and lower for PCL/APIOPOSS containing 5 and $10 \mathrm{wt} \%$ POSS. The crystallization processes of PCL/APIBPOSS nanocomposites are finished in shorter time than that of pure PCL at cooling rates $\geq 10^{\circ} \mathrm{C} \mathrm{min}-1$, while at cooling rates lower than $10^{\circ} \mathrm{C} \mathrm{min}^{-1}, \mathrm{PCL}$ and its nanocomposites exhibit similar $1 / t_{1 / 2}$ values. These results indicate that APIBPOSS nanoparticles could accelerate the crystallization process of PCL, signifying that APIBPOSS acts as nucleation agent; therefore, time needed for crystallization shortens. In the PCL/APIOPOSS nanocomposites, the sample containing $2 \mathrm{wt} \%$ behaves as APIBPOSS-based nanocomposites, whereas in the nanocomposites containing 5 and $10 \mathrm{wt} \%$ APIOPOSS, the crystallization process completion takes a longer time than that of pure PCL. The presence of APIOPOSS at 5 and $10 \mathrm{wt} \%$ loading obstructs the crystal growth process of PCL, hindering the crystallization of PCL. 


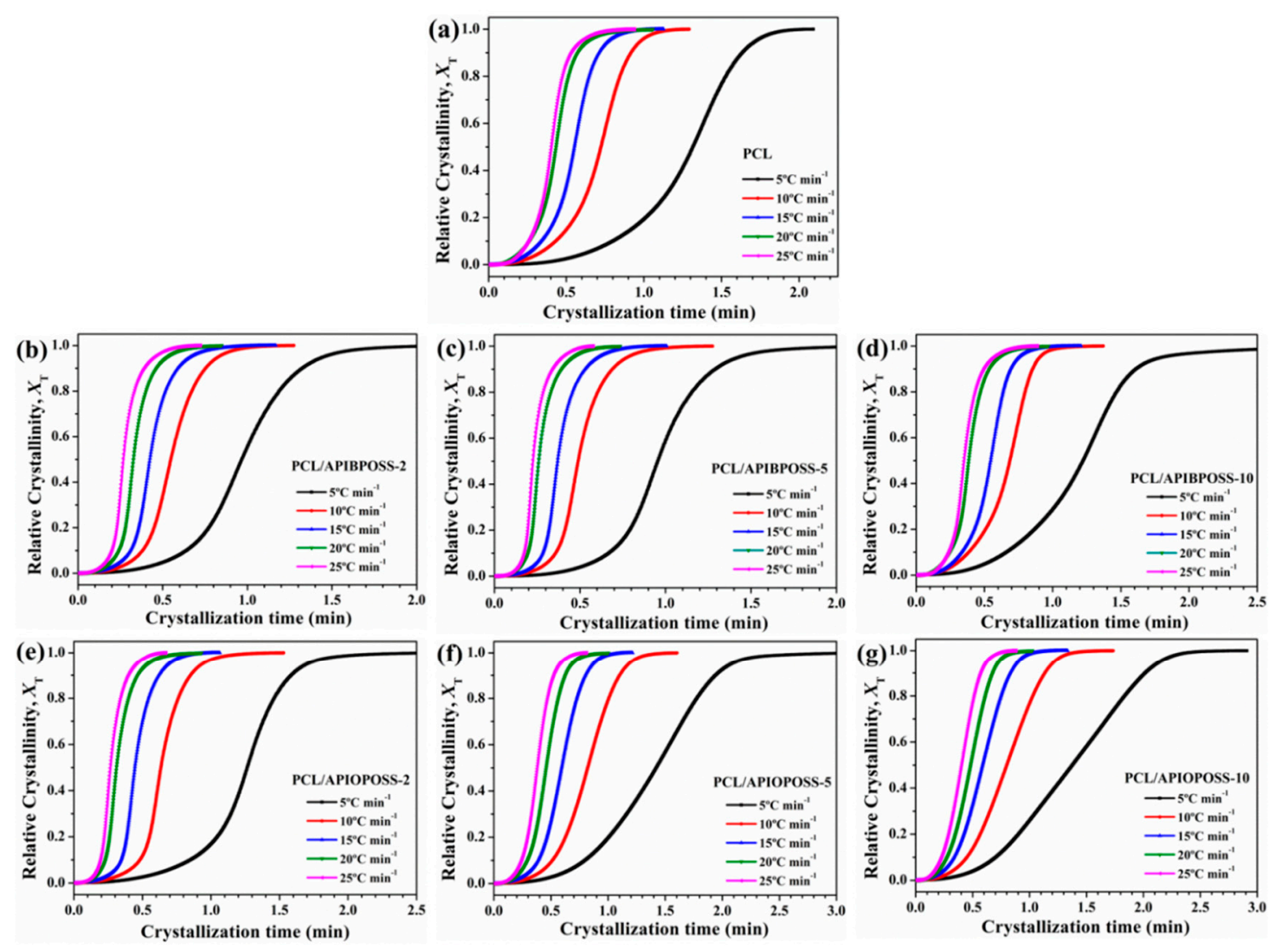

Figure 9. Development of relative crystallinity with time for non-isothermal crystallization of (a) PCL, (b-d) PCL/APIBPOSS nanocomposites, (e-g) PCL/APIOPOSS nanocomposites at various cooling rates.

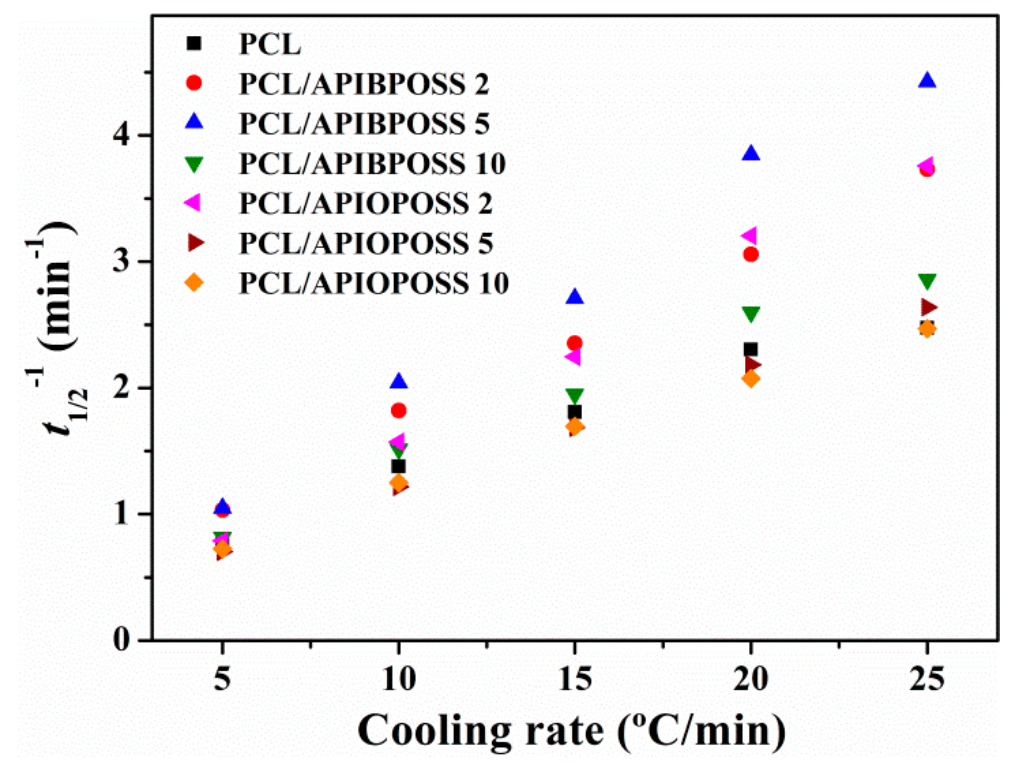

Figure 10. Reciprocal halftime of crystallization at different cooling rates for PCL and PCL/POSS nanocomposites.

In order to quantitatively compare non-isothermal crystallization rates obtained for neat PCL and PCL/POSS nanocomposites, two approaches can be used: (1) the crystallization rate coefficient (CRC) [27] and (2) the crystallization rate parameter (CRP) [28]. The CRC can be determined from the slope of a line by plotting the cooling rate against $T_{\mathrm{m}}-T_{\mathrm{cp}}$. The higher the $\mathrm{CRC}$ value, the faster the crystallization rate. The CRP can be determined from the slope of a line by plotting $1 / t_{1 / 2}$ versus 
cooling rate (Figure 10). The faster the crystallization rate, the higher the slope. The values or CRC and CRP are summarized in Table 1. The CRC value for PCL $\left(3.271 \mathrm{~min}^{-1}\right)$ is lower than that of PCL/POSS nanocomposites containing 2, 5, and $10 \mathrm{wt} \%$ APIBPOSS and 2 and $5 \mathrm{wt} \%$ APIOPOSS (3.806, 4.171, $3.333,4.655$, and $3.511 \mathrm{~min}^{-1}$, respectively), indicating that crystallization rate increases when adding APIBPOSS and APIOPOSS at these concentrations. However, the CRC value of PCL/APIOPOSS-10 nanocomposite is lower than that of $\mathrm{PCL}$, indicating that crystallization rate decreases when adding $10 \%$ APIOPOSS. The CRP value for PCL $\left(0.0854^{\circ} \mathrm{C}^{-1}\right)$ is lower than that of PCL/APIBPOSS nanocomposites (0.1260, 0.1684, and $\left.0.1029{ }^{\circ} \mathrm{C}^{-1}\right)$ and PCL nanocomposites containing 2 and $5 \mathrm{wt} \%$ APIOPOSS $\left(0.1463\right.$ and $\left.0.0897^{\circ} \mathrm{C}^{-1}\right)$ suggesting that these materials are more crystallizable than PCL. The PCL nanocomposite containing $5 \mathrm{wt} \%$ APIBPOSS exhibits the highest CRC and CRP values among the three PCL/APIBPOSS composites, indicating that this content is the most effective in accelerating the crystallization rate of PCL. The nanocomposite containing $2 \mathrm{wt} \%$ APIOPOSS exhibits the highest CRC and CRP values among the three PCL/APIOPOSS composites. Whereas, increasing the APIOPOSS content beyond $5 \mathrm{wt} \%$ retards the crystallization rate. The decrease in crystallization rate with an increase in APIOPOSS content can be attributed to the restrictions imposed by nanoparticles on the mobility of PCL chains, which interfere with the growth of crystals during the crystallization process.

\subsection{Non-Isothermal Crystallization Kinetics}

The non-isothermal crystallization kinetics analysis is of practical importance since it can reflect the crystallization behavior of polymers under processing conditions. Several models have been proposed to study the non-isothermal crystallization of polymers.

\subsubsection{Avrami Analysis}

The Avrami model assumes that crystallization occurs under a constant temperature and expresses the relationship between $X_{t}$ and crystallization time by the following equation:

$$
1-X_{t}=\exp \left(-Z_{t} t^{n}\right)
$$

where $Z_{t}$ is the rate parameter and $n$ is the Avrami exponent, which describes the nucleation (homogeneous or heterogeneous) and growth (rod, disc, sphere, sheaf, etc.) processes in non-isothermal crystallization. Equation (5) is converted to the following equation

$$
\ln \left\{-\ln \left[1-X_{t}\right]\right\}=n \ln t+\ln Z_{t}
$$

In the case of non-isothermal crystallization, nucleation depends on both crystallization time and temperature; however, the Avrami model assumes that only nucleation is a function of crystallization time. To overcome this shortcoming, Jeziorny suggested that the value of rate parameter $Z_{t}$ should be adequately corrected introducing $\phi$ [29]. If $\phi$ is assumed to be constant or approximately constant, the final form of the rate parameter $\left(Z_{c}\right)$ that characterizes the kinetics of non-isothermal crystallization is given as follows:

$$
\ln Z_{c}=\frac{\ln Z_{t}}{\phi}
$$

Drawing the straight line corresponding to $\ln \left\{-\ln \left[1-X_{\mathrm{t}}\right]\right\}$ versus $\ln t$, the value of the Avrami exponent $n$ and the rate parameter $Z_{t}$ can be determined from the slope and the intercept, respectively. Figure 11 shows the double logarithm plots for the non-isothermal process of the PCL and PCL/POSS nanocomposites, respectively. 

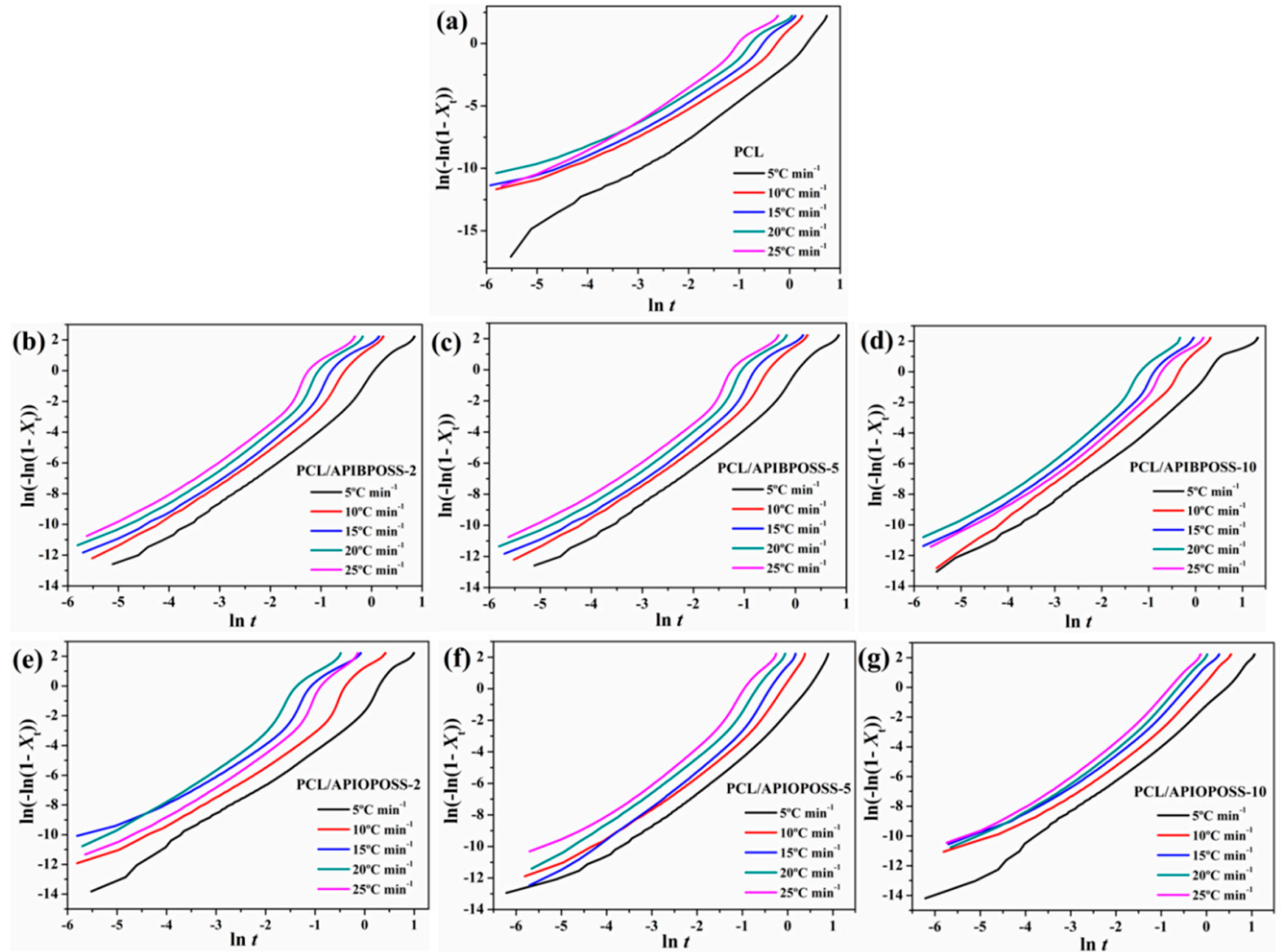

Figure 11. Avrami plots of $\ln \left[-\ln \left(1-X_{\mathrm{t}}\right)\right]$ versus $\ln t$ for non-isothermal crystallization of (a) neat PCL, (b-d) PCL/APIBPOSS nanocomposites, and (e-g) PCL/APIOPOSS nanocomposites.

The curves of neat PCL and PCL/POSS nanocomposites show similar tendencies and indicate that the crystallization of the samples takes place through two main stages involving the primary and secondary crystallization processes. The primary crystallization is comprised of two different stages, indicating the complex process of the crystallization of PCL/POSS nanocomposites.

Different values of $n$ and the rate parameter $\left(Z_{c}\right)$ for the various cooling rates and different stages of crystallization have been determined and compiled in Table 2.

It must be taken into account that in non-isothermal crystallization, the values of $Z_{c}$ and $n$ do not have the same respective physical meaning as in isothermal crystallization, due to the constant change of temperature under non-isothermal conditions. Nevertheless, these values can provide an insight into the kinetics of non-isothermal crystallization for PCL and its POSS-based nanocomposites. $n_{1}$ and $Z_{\mathrm{c} 1}$, and $n_{2}$ and $Z_{\mathrm{c} 2}$ correspond to the first stage of the primary crystallization (which corresponds to a relative conversion of $1-30 \%$, being a little different for each curve), and to the second one (which corresponds to a relative conversion between 30 and $80 \%$, being a little different for each curve), respectively. During the first stage of the primary crystallization, all $n$ were more than 2 (Table 2), indicating that the crystallization growth likely occurred between two-dimensional and three-dimensional patterns. For PCL, all $n_{1}$ values are between 2.5 and 3 , and are not much affected by the presence of amino-POSS. During the second stage, the high $n_{2}$ values $(>3)$ may be due to the combination of other complex processes. $n_{2}$ values are higher than $n_{1}$ values, suggesting that more complicated nucleation mechanism and geometry could be predominant as the crystallization process proceeds. In the case of PCL, $n_{2}$ values are not significantly affected by the cooling rate. 
Table 2. Non-isothermal crystallization kinetics based on Avrami analysis for PCL and PCL/POSS nanocomposites.

\begin{tabular}{|c|c|c|c|c|c|c|c|}
\hline Sample & $\phi\left({ }^{\circ} \mathrm{C} \min ^{-1}\right)$ & $n_{1}$ & $Z_{\mathrm{c} 1}$ & $\mathbf{r}^{2}$ & $n_{2}$ & $Z_{\mathrm{c} 2}$ & $r^{2}$ \\
\hline \multirow{5}{*}{ PCL } & 5 & 3.06 & 0.735 & 0.999 & 5.38 & 0.694 & 0.999 \\
\hline & 10 & 2.56 & 0.999 & 0.997 & 5.38 & 1.148 & 0.999 \\
\hline & 15 & 2.81 & 1.058 & 0.998 & 5.47 & 1.212 & 0.999 \\
\hline & 20 & 2.45 & 1.066 & 0.998 & 5.20 & 1.220 & 0.998 \\
\hline & 25 & 3.07 & 1.081 & 0.999 & 5.36 & 1.213 & 0.999 \\
\hline \multirow{5}{*}{ PCL/APIBPOSS-2 } & 5 & 2.67 & 0.805 & 0.995 & 4.67 & 0.950 & 0.997 \\
\hline & 10 & 2.67 & 1.027 & 0.993 & 5.05 & 1.299 & 0.993 \\
\hline & 15 & 2.78 & 1.066 & 0.993 & 6.03 & 1.372 & 0.991 \\
\hline & 20 & 2.71 & 1.079 & 0.994 & 6.55 & 1.411 & 0.989 \\
\hline & 25 & 2.57 & 1.073 & 0.993 & 7.02 & 1.425 & 0.993 \\
\hline \multirow{5}{*}{ PCL/APIBPOSS-5 } & 5 & 2.94 & 0.803 & 0.995 & 4.54 & 0.966 & 0.993 \\
\hline & 10 & 2.74 & 1.046 & 0.989 & 5.92 & 1.462 & 0.991 \\
\hline & 15 & 2.84 & 1.082 & 0.990 & 7.01 & 1.549 & 0.991 \\
\hline & 20 & 2.80 & 1.117 & 0.987 & 6.90 & 1.563 & 0.989 \\
\hline & 25 & 2.88 & 1.110 & 0.988 & 7.60 & 1.551 & 0.989 \\
\hline \multirow{5}{*}{ PCL/APIBPOSS-10 } & 5 & 2.48 & 0.624 & 0.997 & 3.95 & 0.804 & 0.995 \\
\hline & 10 & 2.55 & 1.023 & 0.999 & 5.51 & 1.212 & 0.998 \\
\hline & 15 & 2.77 & 1.057 & 0.998 & 6.67 & 1.252 & 0.993 \\
\hline & 20 & 2.59 & 1.069 & 0.998 & 5.22 & 1.256 & 0.991 \\
\hline & 25 & 2.68 & 1.061 & 0.993 & 5.67 & 1.247 & 0.993 \\
\hline \multirow{5}{*}{ PCL/APIOPOSS-2 } & 5 & 2.45 & 0.688 & 0.996 & 6.27 & 0.690 & 0.998 \\
\hline & 10 & 2.44 & 0.942 & 0.992 & 7.74 & 1.367 & 0.997 \\
\hline & 15 & 2.74 & 1.036 & 0.993 & 7.62 & 1.472 & 0.995 \\
\hline & 20 & 2.30 & 1.040 & 0.990 & 6.26 & 1.414 & 0.995 \\
\hline & 25 & 2.43 & 1.055 & 0.992 & 6.15 & 1.364 & 0.997 \\
\hline \multirow{5}{*}{ PCL/APIOPOSS-5 } & 5 & 2.55 & 0.711 & 0.995 & 3.40 & 0.738 & 0.999 \\
\hline & 10 & 2.70 & 0.951 & 0.995 & 4.23 & 1.047 & 0.999 \\
\hline & 15 & 2.66 & 0.998 & 0.997 & 4.60 & 1.125 & 0.999 \\
\hline & 20 & 2.51 & 1.032 & 0.997 & 4.50 & 1.165 & 0.999 \\
\hline & 25 & 2.62 & 1.047 & 0.998 & 4.59 & 1.163 & 0.999 \\
\hline \multirow{5}{*}{ PCL/APIOPOSS-10 } & 5 & 2.67 & 0.770 & 0.998 & 2.69 & 0.785 & 0.999 \\
\hline & 10 & 2.75 & 0.996 & 0.997 & 3.29 & 1.038 & 0.999 \\
\hline & 15 & 2.74 & 1.052 & 0.997 & 3.46 & 1.103 & 1.000 \\
\hline & 20 & 2.81 & 1.070 & 0.998 & 3.55 & 1.117 & 1.000 \\
\hline & 25 & 2.75 & 1.076 & 0.998 & 3.47 & 1.118 & 1.000 \\
\hline
\end{tabular}

\subsubsection{Combined Ozawa-Avrami Method}

Ozawa [30] extended the Avrami equation to the non-isothermal condition by replacing the time variable by a cooling rate and derived a kinetic equation as follows:

$$
1-X_{t}=\exp \left[\frac{K(T)}{\phi^{m}}\right]
$$

where $X_{\mathrm{t}}$ is the relative crystallinity, $K(T)$ represents the cooling function, which is related to the overall crystallization rate and indicates the speed at which crystallization occurs, $\phi$ is the cooling rate, and $m$ is the Ozawa exponent depending on the crystal growth and nucleation mechanism. Ozawa assumes that there is no secondary nucleation kinetics and no volume changes during the crystallization process.

Mo and coworkers [31] suggested a novel kinetic model by combining the Avrami Equation (5) with the Ozawa Equation (8):

$$
\ln \phi=\ln F(T)-\alpha \ln t
$$


where the parameter $F(T)=[K(T) / K]^{1 / m}$ refers to the value of the cooling rate chosen at a unit crystallization time, when the system has a certain degree of crystallinity. The smaller the value of $F(T)$, the higher the crystallization rate. The parameter $\alpha$ is the ratio of the Avrami exponent $n$ to Ozawa exponent $m(\alpha=n / m)$. According to Equation (9), a straight line with an intercept of $\ln F(T)$ and a slope of $-\alpha$ should be obtained by plotting $\ln \phi$ against $\ln t$, at a given degree of crystallinity. As it is shown in Figure 12, plotting $\ln \phi$ versus $\ln t$, at a given degree of crystallinity, yields a good relationship. The values of $F(T), \alpha$, and correlation coefficients $\mathrm{r}^{2}$ are listed in Table 3.
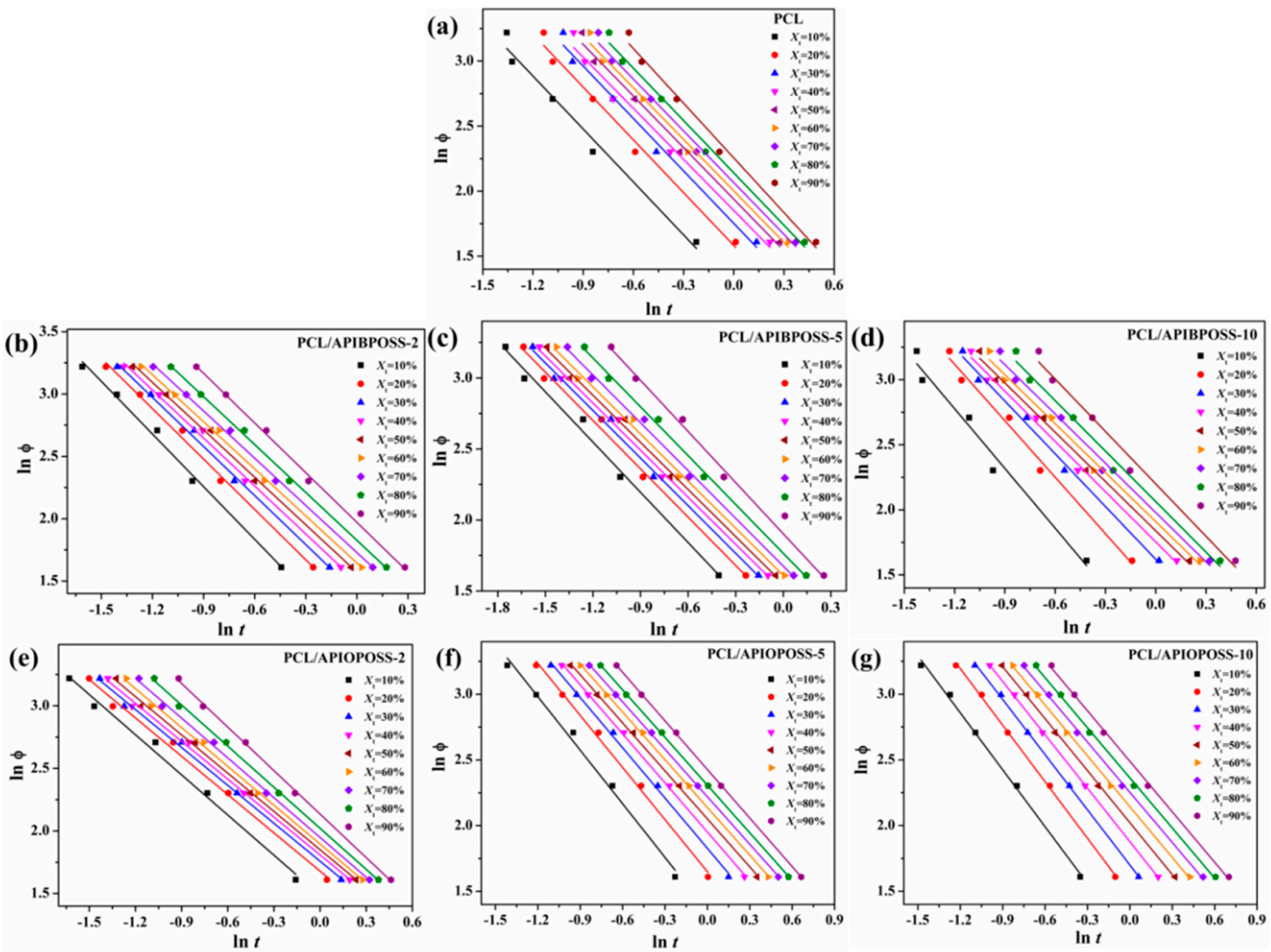

Figure 12. Plots of $\ln \phi$ versus $\ln t$ during the non-isothermal crystallization process of (a) PCL, (b) PCL/APIBPOSS-2, (c) PCL/APIBPOSS-5, (d) PCL/APIBPOSS-10, (e) PCL/APIOPOSS-2,

(f) PCL/APIOPOSS-5, and (g) PCL/APIOPOSS-10 at different relative degree of crystallinity.

The values of $F(T)$ increases with increasing the relative crystallinity, indicating that at unit crystallization time, a higher cooling rate should be used to obtain a higher degree of crystallinity. In addition, the value of $F(T)$ for all PCL/APIBPOSS and PCL/APIOPOSS-2 nanocomposites are generally smaller than those for neat $\mathrm{PCL}$, suggesting that crystallization rate of nanocomposites containing APIBPOSS and $2 \mathrm{wt} \%$ of APIOPOSS is higher than that of PCL. However, values of $F(T)$ for PCL/APIOPOSS nanocomposites containing 5 and $10 \mathrm{wt} \%$ POSS are slightly higher than that of neat $\mathrm{PCL}$, indicating that the crystallization rate for these nanocomposites is slower than that for PCL. These results are in good agreement with those obtained from $t_{1 / 2}$. These results suggest that the presence of APIBPOSS nanoparticles acts as nucleating sites for the dynamic crystallization process of PCL. The values of $\alpha$ show a slight variation at different relative crystallinities for PCL and its nanocomposites, indicating that the method of Mo and co-workers can effectively describe the non-isothermal crystallization kinetic of PCL and PCL/POSS nanocomposites. The advantage of this method is that it correlates the cooling rate to temperature, time, and morphology, that is, nucleation and growth mechanism of crystals. 
Table 3. The non-isothermal crystallization kinetic parameters based on Mo model for PCL and PCL/POSS nanocomposites.

\begin{tabular}{|c|c|c|c|c|c|c|c|c|c|c|}
\hline Sample & $X_{t}(\%)$ & 10 & 20 & 30 & 40 & 50 & 60 & 70 & 80 & 90 \\
\hline PCL & $F(T)$ & 3.39 & 4.77 & 5.68 & 6.30 & 6.85 & 7.32 & 7.81 & 8.42 & 9.42 \\
\hline \multirow[b]{2}{*}{ PCL/APIBPOSS-2 } & $\alpha$ & 1.44 & 1.38 & 1.34 & 1.31 & 1.29 & 1.29 & 1.29 & 1.31 & 1.37 \\
\hline & $F(T)$ & 2.58 & 3.43 & 3.93 & 4.34 & 4.69 & 5.07 & 5.52 & 6.10 & 7.05 \\
\hline \multirow[t]{2}{*}{ PCL/APIBPOSS-5 } & $F(T)$ & 3.09 & 3.79 & 4.15 & 4.43 & 4.68 & 4.95 & 5.29 & 5.77 & 6.61 \\
\hline & $r^{2}$ & 0.9907 & 0.9928 & 0.9935 & 0.9938 & 0.9951 & 0.9948 & 0.9956 & 0.9962 & 0.9953 \\
\hline \multirow{3}{*}{ PCL/APIBPOSS-10 } & $\alpha$ & 1.53 & 1.43 & 1.33 & 1.28 & 1.26 & 1.26 & 1.28 & 1.32 & 1.38 \\
\hline & $F(T)$ & 2.53 & 3.82 & 5.09 & 5.78 & 6.27 & 6.70 & 7.15 & 7.77 & 8.88 \\
\hline & $\mathrm{r}^{2}$ & 0.9814 & 0.9889 & 0.9933 & 0.9947 & 0.9946 & 0.9933 & 0.9927 & 0.9917 & 0.9896 \\
\hline \multirow{3}{*}{ PCL/APIOPOSS-5 } & $\alpha$ & 1.37 & 1.33 & 1.29 & 1.26 & 1.242 & 1.23 & 1.229 & 1.24 & 1.26 \\
\hline & $F(T)$ & 3.93 & 5.27 & 6.27 & 7.09 & 7.82 & 8.53 & 9.25 & 10.12 & 11.39 \\
\hline & $\mathrm{r}^{2}$ & 0.9916 & 0.9955 & 0.9969 & 0.9982 & 0.9982 & 0.9987 & 0.9987 & 0.9990 & 0.9990 \\
\hline \multirow{3}{*}{ PCL/APIOPOSS-10 } & $\alpha$ & 1.38 & 1.39 & 1.37 & 1.34 & 1.32 & 1.29 & 1.28 & 1.28 & 1.29 \\
\hline & $F(T)$ & 3.39 & 4.65 & 5.71 & 6.72 & 7.70 & 8.67 & 9.66 & 10.76 & 13.33 \\
\hline & $r^{2}$ & 0.9897 & 0.9937 & 0.9955 & 0.9967 & 0.9970 & 0.9972 & 0.9970 & 0.9970 & 0.9966 \\
\hline
\end{tabular}

\subsubsection{Crystallization Activation Energy}

For a process that occurs on cooling, reliable values of the effective energy barrier for the process can be obtained by the differential iso-conversional method of Friedman [32]. The Friedman equation is expressed as:

$$
\ln \left(\frac{d X}{d t}\right)_{X, t}=\text { constant }-\frac{\Delta E_{X_{t}}}{R T_{X_{t}}}
$$

where $\mathrm{d} X / \mathrm{d} t$ is the instantaneous crystallization rate as a function of time at a given conversion $X$, and $\Delta E_{X \mathrm{t}}$ is the effective energy barrier of the process at a given conversion $X$. The relative crystallinity function of temperature $X(T)$, obtained from the experimental data shown in Figure 1, needs to be converted to the relative crystallinity function of time $X(\mathrm{t})$ by transforming the horizontal temperature axis into time. Once the function is obtained, it is differentiated with respect to time to obtain $\mathrm{dX} / \mathrm{d} t$. Furthermore, by selecting appropriate degrees of crystallinity, the values of $\mathrm{dX} / \mathrm{d} t$ at a specific $X_{\mathrm{t}}$ are correlated to the corresponding crystallization temperature at this $X_{t}$, i.e., $T_{X t}$. Finally, by plotting the left hand side of Equation (10) with respect to $1 / T_{X t}$, a straight line must be obtained with a slope equal to $\Delta E_{X \mathrm{t}} / R$. The dependence of the effective activation energy $\Delta E_{\mathrm{a}}$ on the relative degree of crystallinity for PCL and PCL/POSS nanocomposites is shown in Figure 13.

The crystallization activation energy is closely related to crystallization process, and can exhibit the crystallization ability; high $\Delta E_{a}$ value implies low crystallization ability. For all the samples studied, the activation energy is negative, indicating that the rate of crystallization increases with decreasing temperature. In the case of PCL, $\Delta E_{a}$ is almost constant up to a $X_{\mathrm{t}}$ of $30 \%$, and then increases with the degree of crystallinity up to an $X_{t}$ of $80 \%$, where the activation energy value attains a maximum value, and then decreases slightly as $X_{t}$ further increases. The $\Delta E_{a}$ values for PCL crystallization were between -140 and $-67 \mathrm{~kJ} \mathrm{~mol}^{-1}$ in the $X_{\mathrm{t}}$ range from 10 to $90 \%$, which are similar to those reported in the literature and obtained by the Friedman isoconversional method [33-35]. $\Delta E_{a}$ value for PCL/APIBPOSS containing 2 and $5 \mathrm{wt} \%$ POSS increases monotonically with increasing the extent of the relative crystallization $\left(X_{\mathrm{t}}\right)$, indicating that crystallization occurs much easier at lower relative crystallinity. However, the composite containing $10 \mathrm{wt} \%$ APIBPOSS decreases as $X_{\mathrm{t}}$ increases from 
10 to $30 \%$, then remains constant in the $X_{t}$ range from 30 to $70 \%$, and finally increases notably as $X_{t}$ further increases. PCL/APIBPOSS composites show lower activation energy than that of neat PCL for a conversion range between 10 and 70\%, indicating that the crystallization ability of PCL in these nanocomposites is higher than neat PCL, due to heterogeneous nucleation effect in the beginning of the process. For PCL/APIOPOSS nanocomposites, only the sample containing $2 \mathrm{wt} \%$ POSS exhibits lower $\Delta E_{a}$ values than neat PCL in the relative crystallinity range from 10 to $60 \%$, whereas activation energy values of those composites with 5 and $10 \mathrm{wt} \%$ APIOPOSS are lower than that of neat PCL in the conversion range from 50 to $90 \%$. The $\Delta E_{a}$ values of PCL/APIBPOSS composites are lower than those of the PCL/APIOPOSS composites, especially in the conversion range from 30 to $70 \%$, indicating that the crystallization ability of PCL in the presence of APIBPOSS is higher than in the presence of APIOPOSS. At low extent of conversion (10-20\%), the composites containing 2 and $10 \mathrm{wt} \%$ APIBPOSS exhibit higher $\Delta E_{a}$ values than those of the composites containing $5 \mathrm{wt} \%$ APIBPOSS and $2 \mathrm{wt} \%$ APIOPOSS, which indicates that the first two need higher effective activation energy for initiation of crystallization. However, at the extent of conversion higher than $30 \%$, PCL/APIOPOSS-2 displays $\Delta E_{a}$ values higher than those of PCL/APIBPOSS composites, suggesting that the diffusion of the melt chains to the growth front becomes more difficult in the case of the APIOPOSS composite.

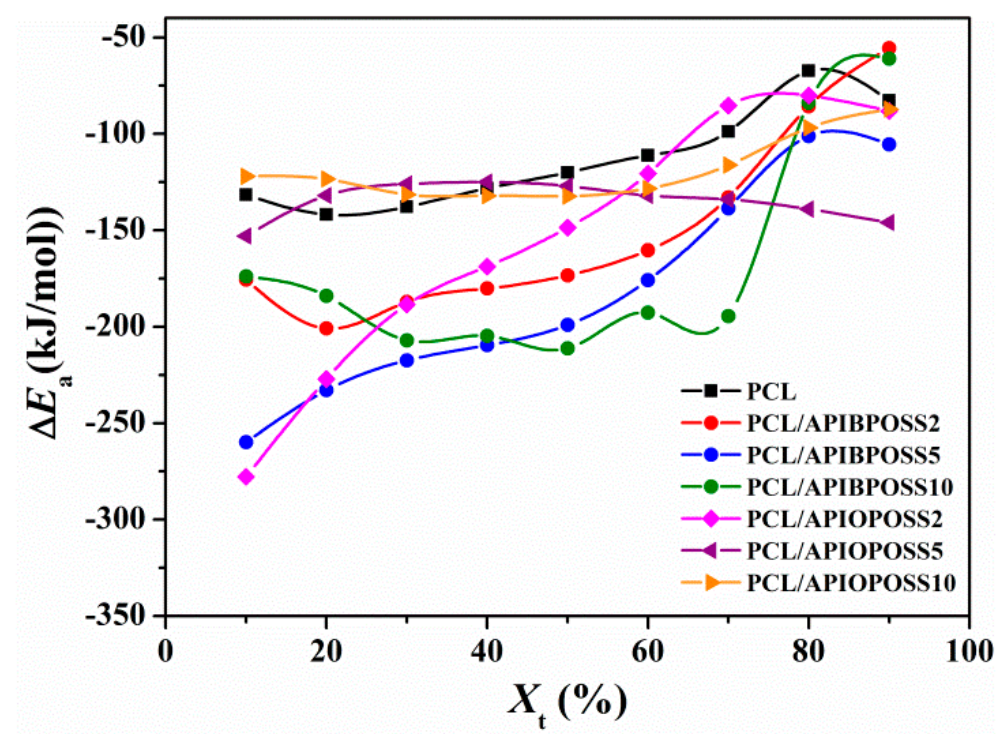

Figure 13. Dependence of the activation energy on the relative crystallinity using isoconversional analysis for PCL and PCL/POSS nanocomposites.

\subsection{XRD Characterization}

Figure 14 shows the XRD patterns of neat PCL and PCL/POSS nanocomposites containing 2 wt $\%$ POSS. Neat PCL and PCL/POSS nanocomposites exhibit the same XRD profile in the $2 \theta$ range of $15-30^{\circ}$, that is, the characteristic diffraction peaks at $2 \theta=21.34^{\circ}, 21.96^{\circ}$, and $23.56^{\circ}$. However, PCL/POSS nanocomposites show weaker diffraction peaks than PCL, and those of nanocomposite containing APIOPOSS are the weakest. This result indicates that the incorporation of POSS nanoparticles has effect on the degree of crystallinity of PCL, and this effect is more pronounced in the presence of APIOPOSS. The addition of APIBPOSS and APIOPOSS leads to a reduction in the extent of crystallinity of polymer matrix, being the reduction more remarkable when the most compatible POSS with PCL is present. This result is in agreement with the DSC results presented in this study. 


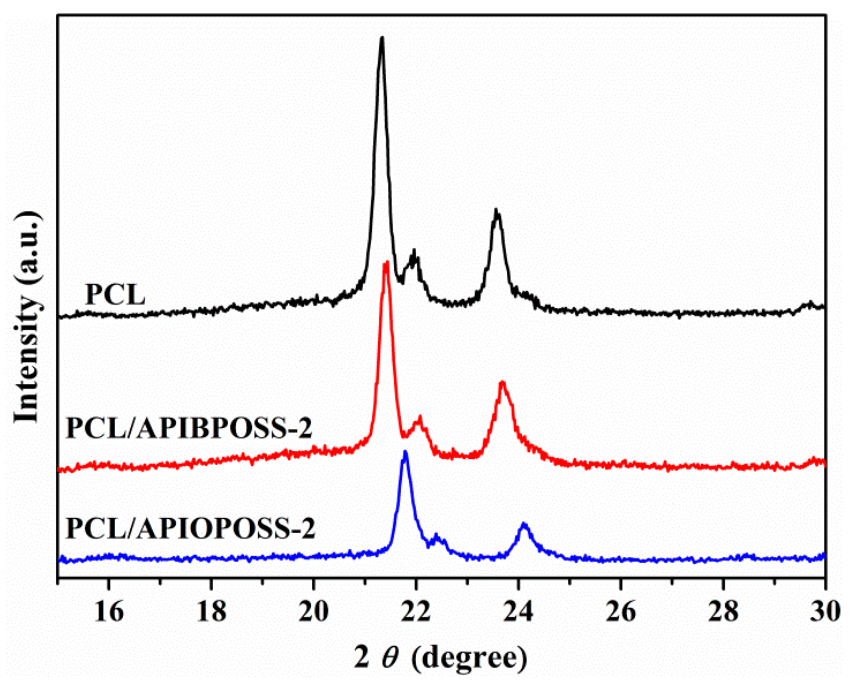

Figure 14. XRD patterns of neat PCL and PCL/POSS nanocomposites.

\subsection{Spherulitic Morphology}

Morphology during non-isothermal crystallization was studied with a polarizing microscope. Polarized micrographs of neat PCL and its nanocomposites after non-isothermal crystallization from 100 to $25^{\circ} \mathrm{C}$ at a cooling rate of $2{ }^{\circ} \mathrm{C} \mathrm{min}^{-1}$ are shown in Figure 15 . The spherulites of neat PCL are fairly larger with the maltese cross than those of the PCL/POSS nanocomposites. Compared with pure PCL, the spherulitic concentration in PCL/POSS nanocomposites is much higher, and the spherulites are smaller and more imperfect. This is attributed to the larger number of heterogeneous nuclei in PCL matrix in the presence of POSS, which reduces the sizes of spherulites.

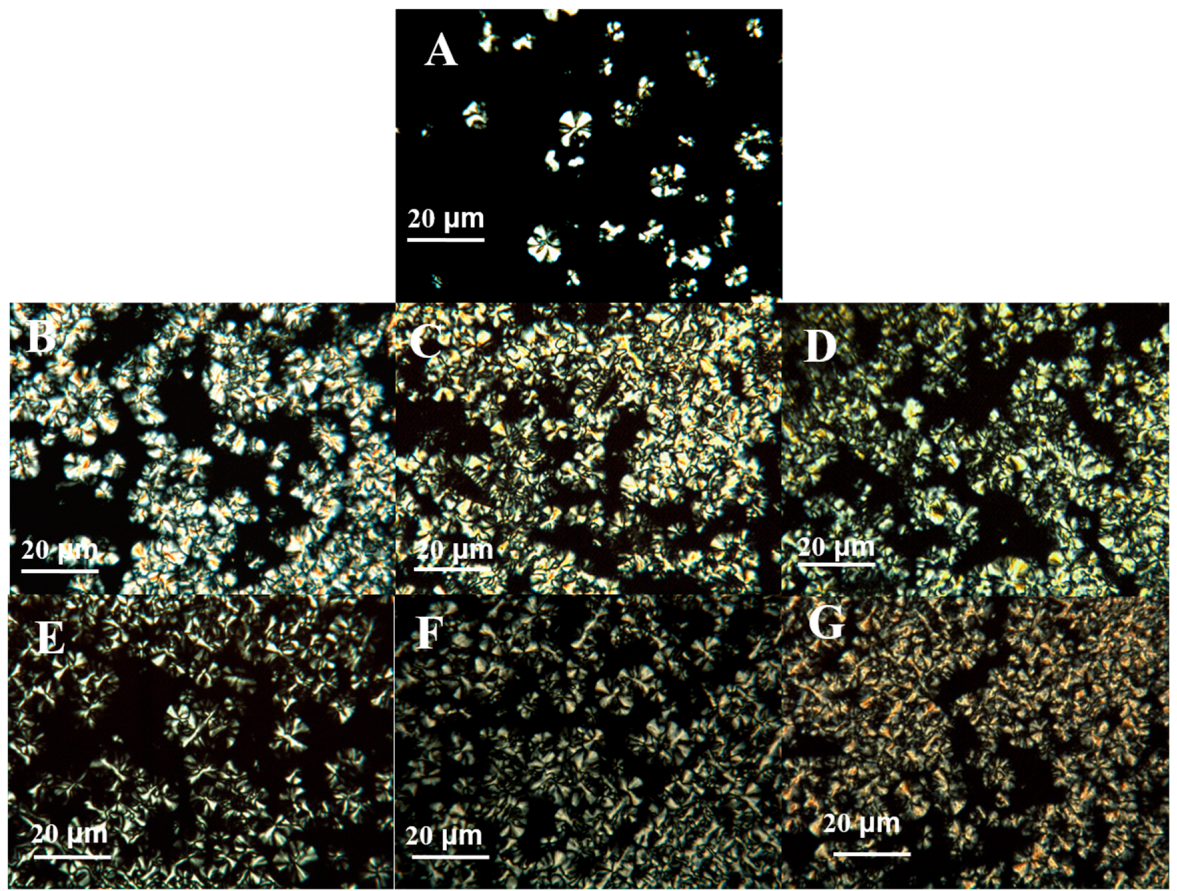

Figure 15. POM images of (A) PCL, (B) PCL/APIBPOSS-2, (C) PCL/APIBPOSS-5, (D) PCL/APIBPOSS-10, (E) PCL/APIOPOSS-2, (F) PCL/APIOPOSS-5, and (G) PCL/APIOPOSS-10 nanocomposites, at $38^{\circ} \mathrm{C}$ during non-isothermal crystallization from their melts at a cooling rate of $2{ }^{\circ} \mathrm{C} \mathrm{min}-1$. 


\section{Conclusions}

Non-isothermal crystallization behavior and kinetics, and subsequent melting behavior of neat PCL, PCL/APIBPOSS, and PCL/APIOPOSS composites containing 2, 5, and $10 \mathrm{wt} \%$ POSS were studied by DSC. The results showed that the crystallization process was affected by the POSS type and loading. POSS hindered the crystallization of PCL due to the confinement caused by the nanoparticles on the PCL. The crystallization peak temperature of PCL shifted to higher temperature and the crystallization rate increased upon incorporation of APIBPOSS at all nanoparticle loadings studied, and at $2 \mathrm{wt} \%$ APIOPOSS. Based on the CRC and CRP approaches, the highest crystallization rate was obtained by addition of $5 \mathrm{wt} \%$ APIBPOSS and $2 \mathrm{wt} \%$ APIOPOSS. The Avrami and combined Ozawa-Avrami methods were able to describe the non-isothermal crystallization process of PCL/POSS nanocomposites. The activation energy for non-isothermal melt crystallization of neat PCL and PCL/POSS composites was determined using Friedman isoconversional method. The addition of APIBPOSS caused a decrease of $\triangle E$. PCL/APIOPOSS composites required more energy for crystallization than PCL/APIBPOSS samples. PCL/POSS nanocomposites with 2, 5, and $10 \mathrm{wt} \%$ of APIBPOSS loading, and $2 \mathrm{wt} \%$ of APIOPOSS loading crystallized easier than PCL. Morphological analysis using POM showed that both APIBPOSS and APIOPOSS dispersed in the PCL matrix acted as a heterogeneous nucleation centers for the crystallization of PCL. The incorporation of POSS nanoparticles into the PCL matrix enhanced the nucleation density of PCL and increased with an increase in the POSS content. POSS nanoparticles played a dual role in the PCL crystallization process: first they served as a nucleating medium to promote the nucleation process of PCL, and second they hindered the mobility of PCL chains.

Author Contributions: Conceptualization, M.D.F. and M.J.F.; formal analysis and interpretation, M.D.F. and M.J.F.; funding acquisition, M.D.F. and M.J.F.; investigation, M.D.F., D.J.G., J.R.R., and M.J.F.; methodology, M.D.F. and M.J.F.; writing—original draft, M.D.F. and M.J.F.; writing—review and editing, M.D.F. and M.J.F. All authors have given approval to the final version of the manuscript.

Funding: This research was funded by the Basque Government (SAIOTEK 2012 S-PE12UN006) and the University of the Basque Country (UFI11/56).

Acknowledgments: Technical and human support provided by SGIker of UPV/EHU and European funding (ERDF and ESF) is gratefully acknowledged.

Conflicts of Interest: The authors declare no conflict of interest.

\section{References}

1. Woodruff, M.A.; Hutmacher, D.W. The return of a forgotten polymer-Polycaprolactone in the 21st century. Prog. Polym. Sci. 2010, 35, 1217-1256. [CrossRef]

2. Kweon, H.Y.; Yoo, M.K.; Park, I.K.; Kim, T.H.; Lee, H.C.; Lee, H.S.; Oh, J.S.; Akaike, T.; Cho, C.S. A novel degradable polycaprolactone networks for tissue engineering. Biomaterials 2003, 24, 801-808. [CrossRef]

3. Messersmith, P.B.; Giannelis, E.P. Synthesis and barrier properties of poly( $\varepsilon$-caprolactone)-layered silicate nanocomposites. J. Polym. Sci. Polym. Chem. 1995, 33, 1047-1057. [CrossRef]

4. Fukushima, K.; Tabuani, D.; Camino, G. Nanocomposites of PLA and PCL based on montmorillonite and sepiolite. Mat. Sci. Eng. C-Mater. 2009, 29, 1433-1441. [CrossRef]

5. Di, Y.; Iannac, S.; Sanguigno, L.; Nicolais, L. Barrier and mechanical properties of poly(caprolactone)/ organoclay nanocomposites. Macromol. Symp. 2005, 228, 115-124. [CrossRef]

6. Chrissafis, K.; Antoniadis, G.; Paraskevopoulos, K.; Vassiliou, A.; Bikiaris, D. Comparative study of the effect of different nanoparticles on the mechanical properties and thermal degradation mechanism of in situ prepared poly( $\varepsilon$-caprolactone) nanocomposites. Compos. Sci. Technol. 2007, 67, 2165-2174. [CrossRef]

7. Zhang, J.; Qiu, Z. Morphology, crystallization behavior and dynamic mechanical properties of biodegradable poly(E-caprolactone)/thermally reduced graphene nanocomposites. Ind. Eng. Chem. Res. 2011, 50, 13885-13891. [CrossRef]

8. Cordes, D.B.; Lickiss, P.D.; Rataboul, F. Recent developments in the chemistry of cubic polyhedral oligosilsesquioxanes. Chem. Rev. 2010, 110, 2081-2173. [CrossRef] 
9. Qian, Q.; Xu, J.; Zhang, M.; He, J.; Ni, P. Versatile Construction of Single-Tailed Giant Surfactants with Hydrophobic Poly( $\varepsilon$-caprolactone) Tail and Hydrophilic POSS Head. Polymers 2019, 11, 311. [CrossRef]

10. Li, Y.; Dong, X.H.; Zou, Y.; Wang, Z.; Yue, K.; Huang, M.; Liu, H.; Feng, X.; Lin, Z.; Zhang, W.; et al. Polyhedral oligomeric silsesquioxane meets "click" chemistry: Rational design and facile preparation of functional hybrid materials. Polymer 2017, 125, 303-329. [CrossRef]

11. Fina, A.; Tabuani, D.; Frache, A.; Camino, G. Polypropylene-polyhedral oligomeric silsesquioxanes (POSS) nanocomposites. Polymer 2005, 46, 7855-7866. [CrossRef]

12. Pan, H.; Yu, J.; Qiu, Z. Crystallization and morphology studies of biodegradable poly(e-caprolactone)/ polyhedral oligomeric silsesquioxanes nanocomposites. Polym. Eng. Sci. 2011, 51, 2159-2165. [CrossRef]

13. Miltner, H.E.; Watzeels, N.; Gotzen, N.A.; Goffin, A.L.; Duquesne, E.; Benali, S.; Ruelle, B.; Peeterbroeck, S.; Dubois, P.; Goderis, B.; et al. The effect of nano-sized filler particles on the crystalline-amorphous interphase and thermal properties in polyester nanocomposites. Polymer 2012, 53, 1494-1506. [CrossRef]

14. Goffin, A.L.; Duquesne, E.; Raquez, J.M.; Miltner, H.E.; Ke, X.; Alexandre, M.; Van Tendeloo, G.; Van Mele, B.; Dubois, P. From polyester grafting onto POSS nanocage by ring-opening polymerization to high performance polyester/POSS nanocomposites. J. Mater. Chem. 2010, 209, 9415-9422. [CrossRef]

15. Miltner, H.E.; Watzeels, N.; Goffin, A.L.; Duquesne, E.; Benali, S.; Dubois, P.; Rahier, H.; Van Mele, B. Quantifying the degree of nanofiller dispersion by advanced thermal analysis: Application to polyester nanocomposites prepared by various elaboration methods. J. Mater. Chem. 2010, 20, 9531-9542. [CrossRef]

16. Guan, W.; Qiu, Z. Isothermal crystallization kinetics, morphology, and dynamic mechanical properties of biodegradable poly( $\varepsilon$-caprolactone) and octavinyl-polyhedral oligomeric silsesquioxanes nanocomposite. Ind. Eng. Chem. Res. 2012, 51, 3203-3208. [CrossRef]

17. Lee, K.S.; Chang, Y.W. Thermal and mechanical properties of poly( $\varepsilon$-caprolactone)/polyhedral oligomeric silsesquioxane nanocomposites. Polym. Int. 2013, 62, 64-70. [CrossRef]

18. Pracella, M.; Chionna, D.; Fina, A.; Tabuani, D.; Frache, A.; Camino, G. Polypropylene-POSS nanocomposites: Morphology and crystallization behaviour. Macromol. Symp. 2006, 234, 59-67. [CrossRef]

19. Baldi, F.; Bignotti, F.; Fina, A.; Tabuani, D.; Riccò, T. Mechanical characterization of polyhedral oligomeric silsesquioxane/polypropylene blends. J. Appl. Polym. Sci. 2007, 105, 935-943. [CrossRef]

20. Heeley, E.L.; Hughes, D.J.; Aziz, Y.E.; Taylor, P.G.; Bassindale, A.R. Morphology and crystallization kinetics of polyethylene/long alkyl-chain substituted polyhedral oligomeric silsesquioxanes (POSS) nanocomposite blends: A SAXS/WAXS study. Eur. Polym. J. 2014, 51, 45-56. [CrossRef]

21. Niemczyk, A.; Dziubek, K.; Sacher-Majewska, B.; Czaja, K.; Dutkiewicz, M.; Marciniec, B. Study of thermal properties of polyethylene and polypropylene nanocomposites with long alkyl chain-substituted POSS fillers. J. Therm. Anal. Calorim. 2016, 125, 1287-1299. [CrossRef]

22. Cobos, M.; Ramos, J.R.; Guzmán, D.J.; Fernández, M.D.; Fernández, M.J. PCL/POSS nanocomposites: Effect of POSS derivative and preparation method on morphology and properties. Polymers 2019, 11, 33. [CrossRef] [PubMed]

23. Kim, J.Y.; Park, H.S.; Kim, S.H. Unique nucleation of multi-walled carbon nanotube and poly(ethylene 2,6-naphthalate) nanocomposites during non-isothermal crystallization. Polymer 2006, 47, 1379-1389. [CrossRef]

24. Gupta, A.K.; Gupta, V.B.; Peters, R.H.; Harland, W.G.; Berry, J.P. The effect of addition of high-density polyethylene on the crystallization and mechanical properties of polypropylene and glass-fiber-reinforced polypropylene. J. Appl. Polym. Sci. 1982, 27, 4669-4686. [CrossRef]

25. Gupta, A.K.; Purwar, S.N. Crystallization of PP in PP/SEBS blends and its correlation with tensile properties. J. Appl. Polym. Sci. 1984, 29, 1595-1609. [CrossRef]

26. Crescenzi, V.; Manzini, G.; Calzolari, G.; Borri, C. Thermodynamics of fusion of poly- $\beta$-propiolactone and poly- $\epsilon$-caprolactone. Comparative analysis of the melting of aliphatic polylactone and polyester chains. Eur. Polym. J. 1972, 8, 449-463. [CrossRef]

27. Khanna, Y.P. A barometer of crystallization rates of polymeric materials. Polym. Engng. Sci. 1990, 30, 1615-1619. [CrossRef]

28. Zhang, U.; Zheng, H.; Lou, X.; Ma, D. Crystallization characteristics of polypropylene and low ethylene content polypropylene copolymer with and without nucleating agents. J. Appl. Polym. Sci. 1994, 51, 51-56. [CrossRef] 
29. Jeziorny, A. Parameters characterizing the kinetics of the non-isothermal crystallization of poly(ethylene terephthalate) determined by d.s.c. Polymer 1978, 19, 1142-1144. [CrossRef]

30. Ozawa, T. Kinetics of non-isothermal crystallization. Polymer 1971, 12, 150-158. [CrossRef]

31. Liu, T.; Mo, Z.; Wang, S.; Zhang, H. Nonisothermal melt and cold crystallization kinetics of poly(aryl ether ether ketone ketone). Polym. Eng. Sci. 1997, 37, 568-575. [CrossRef]

32. Friedman, H. Kinetics of thermal degradation of char-forming plastics from thermogravimetry application to a phenolic plastic. J. Polym. Sci. C 1964, 6, 183-195. [CrossRef]

33. Limwanich, W.; Phetsuk, S.; Meepowpan, P.; Kungwan, N.; Punyodom, W. Kinetics studies of non-isothermal melt crystallization of poly(E-caprolactone) and poly(L-lactide). Chiang Mai J. Sci. 2016, 43, 329-338.

34. Oliveira Pires, L.S.; Vaz Fernandes, M.H.F.; Marques de Oliveira, J.M. Crystallization kinetics of PCL and PCL-Glass composites for additive manufacturing. J. Therm. Anal. Calorim. 2018, 134, 2115-2125. [CrossRef]

35. Ahmed, J.; Luciano, G.; Schizzi, I.; Arfat, Y.A.; Maggiore, S.; Thai, T.L.A. Non-isothermal crystallization behavior, rheological properties and morphology of poly( $\varepsilon$-caprolactone)/graphene oxide nanosheets composite films. Thermochim. Acta 2018, 659, 96-104. [CrossRef]

(C) 2019 by the authors. Licensee MDPI, Basel, Switzerland. This article is an open access article distributed under the terms and conditions of the Creative Commons Attribution (CC BY) license (http://creativecommons.org/licenses/by/4.0/). 